\title{
Quantum discontinuity fixed point and renormalization group flow of the Sachdev-Ye-Kitaev model
}

\author{
Roman Louis Smit $\odot,{ }^{1}$ Davide Valentinis $\odot,{ }^{2,3}$ Jörg Schmalian $\odot,{ }^{2,3}$ and Peter Kopietz ${ }^{1}$ \\ ${ }^{1}$ Institut für Theoretische Physik, Universität Frankfurt, Max-von-Laue Strasse 1, 60438 Frankfurt, Germany \\ ${ }^{2}$ Institut für Theorie der Kondensierten Materie, Karlsruher Institut für Technologie, 76131 Karlsruhe, Germany \\ ${ }^{3}$ Institut für Quantenmaterialien und Technologien, Karlsruher Institut für Technologie, 76131 Karlsruhe, Germany
}

(Received 7 October 2020; revised 28 June 2021; accepted 29 June 2021; published 23 July 2021)

\begin{abstract}
We determine the global renormalization group (RG) flow of the Sachdev-Ye-Kitaev (SYK) model. From a controlled truncation of the infinite hierarchy of the exact functional RG flow equations, we identify several fixed points. Apart from a stable fixed point, associated with the celebrated non-Fermi liquid state of the model, we find another stable fixed point related to an integer-valence state. These stable fixed points are separated by a discontinuity fixed point with one relevant direction, describing a quantum first-order transition. Most notably, the fermionic spectrum continues to be quantum critical even at the discontinuity fixed point. This rules out a description of the transition in terms of a local effective Ising variable as is established for classical transitions. We propose an entangled quantum state at phase coexistence as a possible physical origin of this critical behavior.
\end{abstract}

DOI: 10.1103/PhysRevResearch.3.033089

\section{INTRODUCTION}

The liquid-vapor transition of real gases is the prime example of a first-order phase transition that preserves the symmetry and terminates at a critical end point [1]. Such transitions are of importance in systems as diverse as hot and dense nuclear matter [2], polymer-gel fluid mixtures [3], and correlated-electron systems. The famous Mott transition between states of localized and delocalized electrons [4-17], the Kondo volume-collapse transition [18-22], or valence transitions in intermetallic compounds [23] are prominent correlated-electron problems of this kind. Significant experimental and theoretical insights have been gained with regards to the behavior of the classical critical end point of these electronic states; it is described in terms of an effective Ising model where the Ising variable labels coarse grained regions of the two phases at the transition. The end point is then governed by critical elasticity, a vanishing bulk modulus, and rich crossover behavior due to the finite shear modulus of crystalline solids [5,6,24-28].

Much less is known about the associated quantum firstorder transition. It is an open question whether the description in terms of an effective Ising model, successful for classical transitions, continues to be appropriate. This is particularly ambiguous if the transition is from a non-Fermi liquid, strange-metal state to a fully localized incompressible state of matter. Exotic behavior was found near several quantum firstorder transitions [29-32]. A symmetry-preserving first-order transition that terminates at a critical end point was recently identified [33-38] in the Sachdev-Ye-Kitaev (SYK) model $[39,40]$ and generalizations thereof. The SYK model proved

Published by the American Physical Society under the terms of the Creative Commons Attribution 4.0 International license. Further distribution of this work must maintain attribution to the author(s) and the published article's title, journal citation, and DOI. important for our understanding of the intriguing properties of interacting quantum matter without quasiparticles [35,41-49]. The ability to perform controlled calculations in the strongcoupling regime of the SYK model makes it a promising platform to elucidate the role of electronic dynamics at a quantum first-order transition.

In this paper, using a functional renormalization group (FRG) approach, we analyze the scaling behavior at a firstorder quantum phase transition in the SYK model. Performing a large- $N$ truncation of the formally exact FRG flow equations [50-54], we derive the global renormalization group ( $R G)$ flow of the SYK model at zero temperature. As shown in Fig. 1, a flow profile emerges that exhibits four different fixed points: Apart from a trivial vacuum fixed point $V$ we find two stable fixed points $S_{+}$and $S_{-}$separated by a discontinuity fixed point $D$. The latter is driven by changing the chemical potential $\mu$ and, as we will show, describes the first-order transition between a critical non-Fermi liquid state $\left(S_{+}\right)$and an integer-valence state $\left(S_{-}\right)$. The equation of state of this transition was analyzed in Refs. [34,37]; similar transitions were discussed in a number of closely related models $[33,35,36]$, reflecting for example a drastic change from fast to slow quantum information scrambling [33]. Here we determine the relevant exponent of the discontinuity fixed point in accordance with the scaling theory of first-order transitions $[55,56]$. In addition, we find that fermions at the transition are governed by quantum-critical dynamics with an anomalous dimension, despite the discontinuous change of thermodynamic variables. Our results are a consequence of the subtle interplay between high-energy and low-energy dynamics that can be captured by the FRG approach developed here.

\section{SYK MODEL}

The SYK model describes $N$ species of fermions interacting with random two-body interactions,

$$
\mathcal{H}=-\mu \sum_{i} c_{i}^{\dagger} c_{i}+\sum_{i<j, k<l} J_{i j, k l} c_{i}^{\dagger} c_{j}^{\dagger} c_{k} c_{l} .
$$




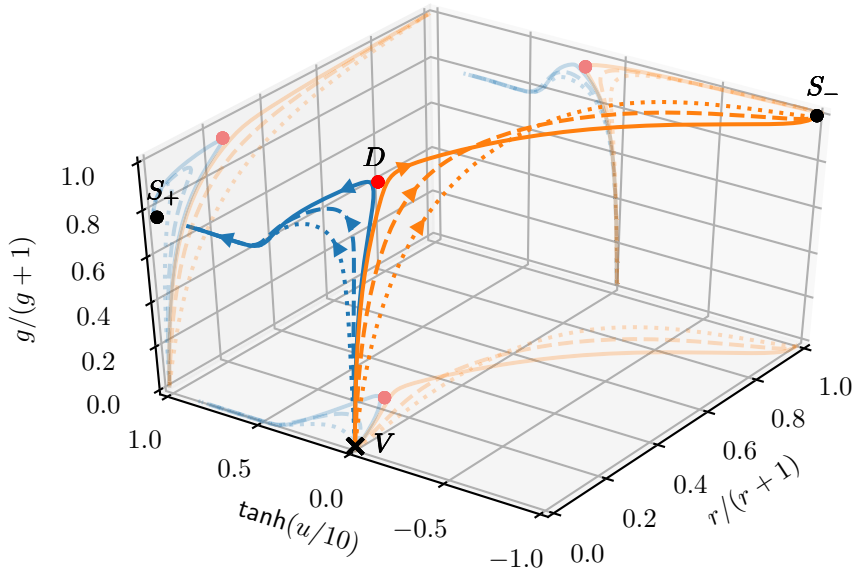

FIG. 1. Global RG flow of the SYK model from Eq. (38). The dimensionless coupling $r$ determines the flow of $\Sigma(0)-\mu$ [where $\Sigma(0)$ is the self-energy at frequency $\omega=0$ and $\mu$ is the chemical potential], while $u$ and $g$ are rescaled four and six-point vertices for vanishing frequencies. At high energies, the flow starts near the vacuum fixed point $V$. The discontinuity fixed point $D$ separates the stable fixed points $S_{+}$and $S_{-}$characterized by non-Fermi liquid and integer-valence behavior, respectively. Most notably, the firstorder transition described by $D$ is characterized by quantum-critical fermions with anomalous dimension $\eta=1 / 2$.

Here $c_{i}$ and $c_{i}^{\dagger}$ are fermionic annihilation and creation operators, $i, j \ldots$ label $N$ different orbitals or lattice sites, and $\mu$ is the chemical potential. The $J_{i j, k l}$ are random variables with Gaussian distribution of zero mean and variance

$$
\overline{\left|J_{i j, k l}\right|^{2}}=2 J^{2} / N^{3} \text {. }
$$

The model is exactly solvable in the limit $N \rightarrow \infty$ where the single-particle properties are determined by the self-energy

$$
\Sigma(\tau)=-J^{2} G^{2}(\tau) G(-\tau)
$$

together with the Dyson equation

$$
G^{-1}(\omega)=i \omega+\mu-\Sigma(\omega) .
$$

The propagator and self-energy are site independent as we refer to the quantities after the averaging over random configurations of the interaction was performed.

At temperature $T=0$, one can construct two solutions of this set of equations. On the one hand, there is the critical, non-Fermi liquid (NFL) solution which takes for $|\omega| \ll J$, the form

$$
\Sigma_{\mathrm{NFL}}(\omega)=\mu-\frac{J A(\theta, \omega)}{\pi^{1 / 4}}\left|\frac{\omega}{J}\right|^{1-\eta},
$$

with anomalous dimension $\eta=1 / 2$ [57]. The coefficient

$$
A=\cos ^{1 / 4}(2 \theta) \operatorname{sign}(\omega) e^{i\left(\theta+\operatorname{sign}(\omega) \frac{\pi}{2}\right)}
$$

depends on the particle number

$$
n=\frac{1}{2}+\frac{\theta}{\pi}+\frac{\sin (2 \theta)}{4}
$$

through the angle $\theta \in\left[-\frac{\pi}{4}, \frac{\pi}{4}\right]$ [41]. The NFL solution, Eq. (5), with power-law propagator $G(\tau) \propto \tau^{-1 / 2}$ yields the much discussed finite ground state entropy, displays analogies

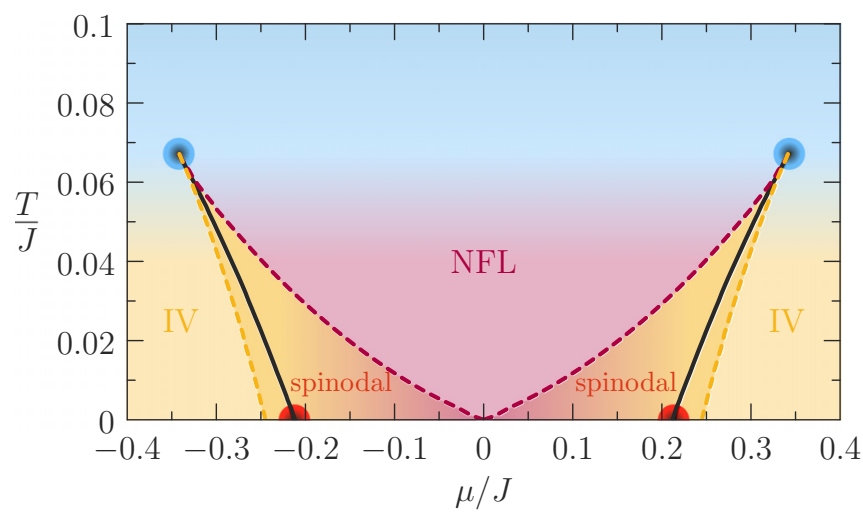

FIG. 2. Phase diagram of the SYK model as a function of chemical potential $\mu$ and temperature $T$ obtained from the numerical solution of the large- $N$ self-consistency equation for the self-energy; see also Ref. [34]. For $T=0$ and $|\mu| \leqslant \mu_{*}=0.212 J$, the non-Fermi liquid (NFL) phase with anomalous dimension $\eta=1 / 2$ is the stable solution. For $\mu= \pm \mu_{*}$ (red dots), there are first-order quantum phase transitions from $n \approx 0.76$ or 0.24 to integer-valence (IV) phases $n=1$ or 0 , respectively. Between the spinodal lines (dashed) both phases are locally stable. The transition terminates at critical points $\left(\mu_{c}, T_{c}\right)=( \pm 0.34,0.067) J$ (blue dots).

to the physics of black holes with regards to its information scrambling, led to theories of the transport properties of strange metals, and allowed for the analysis of superconductivity in non-Fermi liquids [35,41-49]. However, at $T=0$ and finite $\mu$, one can easily show that

$$
\Sigma_{\mathrm{IV}}(\omega)=0
$$

is another solution of the large- $N$ equations describing an integer-valence (IV) state. Depending on the sign of $\mu$ we have $n=1$ or 0 , i.e., a completely filled or empty system. As shown in Ref. [34], a first-order transition occurs between both solutions at $\mu= \pm \mu_{*}(T)$. At $T=0$ and $\mu=$ $\mu_{*}(0) \approx 0.212 J$ the density jumps from $n \approx 0.76$ to 1 . For $\mu=-\mu_{*}(0)$, it jumps from 0.24 to 0 . At finite temperature, $\mu_{*}$ terminates at a critical end point. In Fig. 2, we reproduce the phase diagram already discussed in Ref. [34]. Figure 3 shows the particle number as a function of chemical potential. The discontinuous valence transition below $T_{c}$ and the diverging compressibility at the critical end point are clearly visible.

\section{FUNCTIONAL RENORMALIZATION GROUP}

Identifying the critical surface and the relevant scaling variables should give important additional insights into a model with scale-invariant solutions. Surprisingly, in spite of significant recent attention to the SYK model, we have not been able to find an answer to these fundamental issues in the published literature. In order to derive the FRG flow equations, we perform the average over disorder configurations which yields, in the absence of replica symmetry breaking, an effective four-body interaction with the Euclidean action [41]

$$
S_{8}=-\frac{J^{2}}{N^{3}} \frac{1}{(2 !)^{2}} \int_{0}^{\beta} d \tau \int_{0}^{\beta} d \tau^{\prime}\left|\sum_{i=1}^{N} \bar{c}_{i}(\tau) c_{i}\left(\tau^{\prime}\right)\right|^{4},
$$




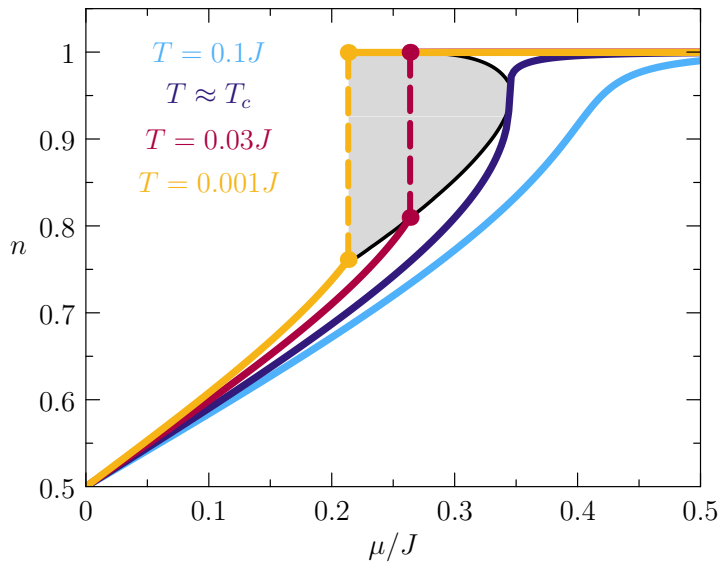

FIG. 3. Particle number $n$ as a function of chemical potential $\mu \geqslant 0$ for different temperatures. For $T<T_{c}$ the density jumps discontinuously across the shaded region.

where $c_{i}(\tau)$ and $\bar{c}_{i}(\tau)$ are Grassmann variables and $\beta=1 / T$. The FRG is then based on introducing a frequency-dependent regulator in the Gaussian part of the action, governed by the cutoff parameter $\Lambda$, which suppresses excitations with energy below $\Lambda$. The physical problem is then recovered in the limit $\Lambda \rightarrow 0$. For our purpose, it suffices to work with a sharp frequency cutoff and use the scale-dependent bare propagator

$$
G_{0, \Lambda}(\omega)=\frac{\Theta(|\omega|-\Lambda)}{i \omega+\mu} .
$$

Following the usual procedure [50-54], we can now derive the Wetterich equation [58] for the scale-dependent effective action $\Gamma_{\Lambda}[\langle\bar{c}\rangle,\langle c\rangle]$, which generates an infinite hierarchy of coupled integro-differential equations for the scale-dependent one-particle irreducible $k$-point vertices $\Gamma_{\Lambda}^{(k)}$. This infinite hierarchy simplifies considerably in the large- $N$ limit. To leading order in $1 / N$, it is sufficient to keep in the flow equation for the $k$-point vertex only diagrams involving the $(k+2)$-point vertex, see Fig. 4. For the flow equation of the self-energy $\Sigma_{\Lambda}(\omega)(k=2)$ this is exact, while for the higher-order vertices the neglected diagrams are subleading in $1 / N$. The bare four-body interaction (9) implies that at the initial scale only the antisymmetrized eight-point vertex $\Gamma_{\Lambda_{0}}^{(8)} \propto-J^{2}$ is finite. To leading order in $1 / N$, the eight-point vertex stays constant throughout the flow. The majority of this section is devoted to the derivation of the flow equations for the rescaled couplings. If the reader is not interested in the technical details, they may want to note the definitions of the rescaled couplings $r_{l}, u_{l}$, and $g_{l}$ given in Eq. (36) and skip to the flow equations (38). To proceed with the derivation, it is convenient to Fourier transform $S_{8}$ given in Eq. (9) to Matsubara frequencies,

$$
\begin{aligned}
S_{8}= & -\frac{J^{2}}{N^{3}} \frac{1}{(2 !)^{2}} \sum_{i j k l=1}^{N} \frac{1}{\beta^{8}} \sum_{\omega_{1}^{\prime} \omega_{2}^{\prime} \omega_{3}^{\prime} \omega_{4}^{\prime}} \sum_{\omega_{1} \omega_{2} \omega_{3} \omega_{4}} \\
& \times \beta \delta_{\omega_{1}^{\prime}+\omega_{2}^{\prime}, \omega_{3}+\omega_{4}} \beta \delta_{\omega_{3}^{\prime}+\omega_{4}^{\prime}, \omega_{1}+\omega_{2}} \\
& \times \bar{c}_{i, \omega_{1}^{\prime}} \bar{c}_{j, \omega_{2}^{\prime}} \bar{c}_{k, \omega_{3}^{\prime}} \bar{c}_{l, \omega_{4}^{\prime}} c_{i, \omega_{1}} c_{j, \omega_{2}} c_{k, \omega_{3}} c_{l, \omega_{4}} .
\end{aligned}
$$

A graphical representation of the interaction vertex in $S_{8}$ is shown in Fig. 5. This expression can be written in an antisym- (a)

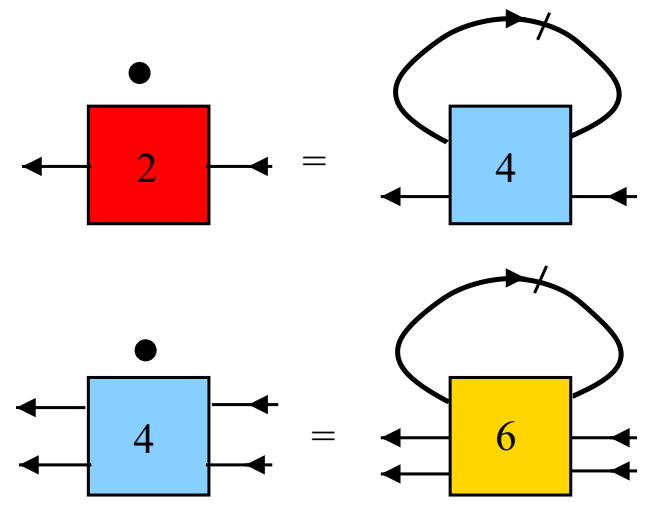

(c)

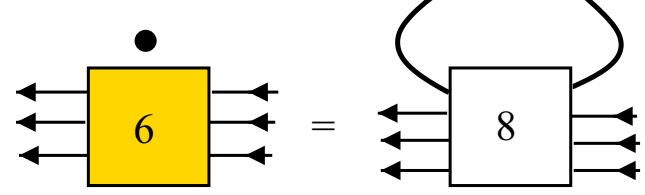

FIG. 4. Graphical representation of the truncated hierarchy of FRG flow equations leading to Eq. (38). The empty square with the label 8 in (c) represents the initial value of the average eight-point vertex given $\propto J^{2}$. Note, that the flow equation (a) for the self-energy is exact, while on the right-hand side of the flow equations (b) and (c) for the four-point and the six-point vertices, we have retained only the contributions from the vertices with the largest number of legs, which give the leading contributions for large $N$.

metrized form as

$$
\begin{aligned}
S_{8}= & \frac{1}{N^{3}} \frac{1}{(4 !)^{2}} \sum_{i^{\prime} j^{\prime} k^{\prime} l^{\prime}} \sum_{i j k l} \frac{1}{\beta^{8}} \sum_{\omega_{1}^{\prime} \omega_{2}^{\prime} \omega_{3}^{\prime} \omega_{4}^{\prime}} \sum_{\omega_{1} \omega_{2} \omega_{3} \omega_{4}} \\
& \times \beta \delta_{\omega_{1}^{\prime}+\cdots+\omega_{4}^{\prime}, \omega_{1}+\cdots+\omega_{4}} \\
& \times \Gamma_{\Lambda_{0} ; i^{\prime} j^{\prime} k^{\prime} l^{\prime} ; i j k l}\left(\omega_{1}^{\prime}, \ldots, \omega_{4}^{\prime} ; \omega_{4}, \ldots, \omega_{1}\right) \\
& \times \bar{c}_{i, \omega_{1}^{\prime}} \bar{c}_{j, \omega_{2}^{\prime}} \bar{c}_{k, \omega_{3}^{\prime}} \bar{c}_{l, \omega_{4}^{\prime}} c_{i, \omega_{1}} c_{j, \omega_{2}} c_{k, \omega_{3}} c_{l, \omega_{4}},
\end{aligned}
$$

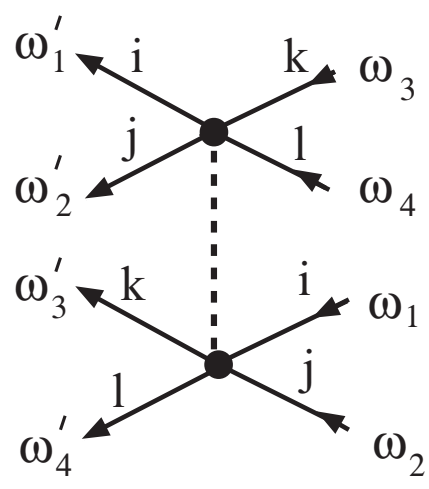

FIG. 5. Graphical representation of the bare interaction vertex in the four-particle interaction $S_{8}$ given in Eq. (11). Outgoing arrows represent $\bar{c}_{n, \omega}$, incoming arrow represent $c_{n, \omega}$, black dots represent factors of $J$ and the dashed line connects the two parts of the vertex where the frequencies are separately conserved. 
which defines the antisymmetrized eight-point vertex

$$
\begin{aligned}
& \Gamma_{\Lambda_{0} ; i^{\prime} j^{\prime} k^{\prime} l^{\prime} ; i j k l}^{(8)}\left(\omega_{1}^{\prime}, \ldots, \omega_{4}^{\prime} ; \omega_{4}, \ldots, \omega_{1}\right) \\
& \quad=-\frac{J^{2}}{(2 !)^{2}} \delta_{i^{\prime}, i} \ldots \delta_{k^{\prime}, k} \beta \delta_{\omega_{1}^{\prime}+\omega_{2}^{\prime}, \omega_{3}+\omega_{4}} \\
& \quad \pm\left[(4 !)^{2}-1 \text { fermionic premutations }\right] .
\end{aligned}
$$

In principle, the self-energy is different for every fermion species, but because of the random interaction the variation around the averaged self-energy becomes arbitrarily small in the limit of large $N$ so that we can write

$$
\Sigma_{\Lambda ; i}(\omega) \approx \frac{1}{N} \sum_{j} \Sigma_{\Lambda ; j}(\omega) \equiv \Sigma_{\Lambda}(\omega) .
$$

To leading order in $1 / N$, only averaged vertices of the form

$$
\begin{aligned}
& \Gamma_{\Lambda}^{(2 k)}\left(\omega_{1}, \ldots, \omega_{k}\right) \\
& \quad \equiv \frac{1}{N^{k}} \sum_{i_{1}, \ldots, i_{k}} \Gamma_{\Lambda ; i_{1} \ldots i_{k} ; i_{1} \ldots i_{k}}^{(2 k)}\left(\omega_{1}, \ldots, \omega_{k} ; \omega_{k}, \ldots, \omega_{1}\right)
\end{aligned}
$$

generate contributions to the averaged self-energy. This leads to the explicit form of the averaged bare antisymmetrized eight-point vertex,

$$
\begin{aligned}
& \Gamma_{\Lambda_{0}}^{(8)}\left(\omega_{1}, \ldots, \omega_{4}\right) \\
& \quad=-2 J^{2} \beta\left[\delta_{\omega_{1}+\omega_{2}, \omega_{3}+\omega_{4}}+\delta_{\omega_{1}+\omega_{3}, \omega_{2}+\omega_{4}}+\delta_{\omega_{1}+\omega_{4}, \omega_{2}+\omega_{3}}\right] .
\end{aligned}
$$

The flow equations represented in Fig. 4 simplify to the averaged flow equations

$$
\begin{aligned}
\partial_{\Lambda} \Sigma_{\Lambda}\left(\omega_{1}\right) & =\frac{1}{\beta} \sum_{\omega_{2}} \dot{G}_{\Lambda}\left(\omega_{2}\right) \Gamma_{\Lambda}^{(4)}\left(\omega_{1}, \omega_{2}\right), \\
\partial_{\Lambda} \Gamma_{\Lambda}^{(4)}\left(\omega_{1}, \omega_{2}\right) & =\frac{1}{\beta} \sum_{\omega_{3}} \dot{G}_{\Lambda}\left(\omega_{3}\right) \Gamma_{\Lambda}^{(6)}\left(\omega_{1}, \omega_{2}, \omega_{3}\right),(17 \mathrm{~b}) \\
\partial_{\Lambda} \Gamma_{\Lambda}^{(6)}\left(\omega_{1}, \omega_{2}, \omega_{3}\right) & =\frac{1}{\beta} \sum_{\omega_{4}} \dot{G}_{\Lambda}\left(\omega_{4}\right) \Gamma_{\Lambda_{0}}^{(8)}\left(\omega_{1}, \omega_{2}, \omega_{3}, \omega_{4}\right),
\end{aligned}
$$

where the single-scale propagator $[52,53]$ is defined as

$$
\dot{G}_{\Lambda}(\omega) \equiv G_{\Lambda}^{2}(\omega) \partial_{\Lambda} G_{0, \Lambda}^{-1}(\omega),
$$

and the scale-dependent dressed propagator is

$$
\begin{aligned}
G_{\Lambda}(\omega) & =\frac{1}{G_{0, \Lambda}^{-1}(\omega)-\Sigma_{\Lambda}(\omega)} \\
& =\frac{\Theta(|\omega|-\Lambda)}{i \omega+\mu-\Sigma_{\Lambda}(\omega)} .
\end{aligned}
$$

Using Morris' lemma [52,59],

$$
\delta(x) f(\Theta(x))=\delta(x) \int_{0}^{1} d t f(t),
$$

the single-scale propagator can be calculated,

$$
\dot{G}_{\Lambda}(\omega)=-\frac{\delta(|\omega|-\Lambda)}{i \omega+\mu-\Sigma_{\Lambda}(\omega)} .
$$

The flow equation (17c) can be integrated straightforwardly by using the explicit form (16) for $\Gamma_{\Lambda_{0}}^{(8)}$ and the Katanin substitution [60]

$$
\dot{G}_{\Lambda} \approx \partial_{\Lambda} G_{\Lambda}
$$

with the result

$$
\begin{aligned}
\Gamma_{\Lambda}^{(6)}\left(\omega_{1}, \omega_{2}, \omega_{3}\right) \\
=-2 J^{2}\left[G_{\Lambda}\left(\omega_{1}+\omega_{2}-\omega_{3}\right)\right. \\
\left.\quad+G_{\Lambda}\left(\omega_{1}-\omega_{2}+\omega_{3}\right)+G_{\Lambda}\left(-\omega_{1}+\omega_{2}+\omega_{3}\right)\right] .
\end{aligned}
$$

A strong argument for the validity of the Katanin substitution in the present context is the fact that repeated substitution leads to the known exact equation (3) for the self-energy of the SYK model; we will show this explicitly in the paragraph leading to Eq. (42). In the limit of zero temperature the Matsubara sums become integrals and the frequencies become continuous. We expand the self-energy at low frequencies as

$$
\Sigma_{\Lambda}(\omega)=\Sigma_{\Lambda}(0)+\left(1-\frac{1}{Z_{\Lambda}}\right) i \omega+\cdots
$$

and rewrite the flow equations (17a) and (17b) at $T=0$ as

$$
\begin{aligned}
\partial_{\Lambda} \Sigma_{\Lambda}(0) & =\int \frac{d \omega_{2}}{2 \pi} \dot{G}_{\Lambda}\left(\omega_{2}\right) \Gamma_{\Lambda}^{(4)}\left(0, \omega_{2}\right), \\
\partial_{\Lambda}\left(-\frac{1}{Z_{\Lambda}}\right) & =\left.\int \frac{d \omega_{2}}{2 \pi} \dot{G}_{\Lambda}\left(\omega_{2}\right) \frac{\partial \Gamma_{\Lambda}^{(4)}\left(\omega_{1}, \omega_{2}\right)}{\partial\left(i \omega_{1}\right)}\right|_{\omega_{1}=0} \\
\partial_{\Lambda} \Gamma_{\Lambda}^{(4)}\left(\omega_{1}, \omega_{2}\right) & =\int \frac{d \omega_{3}}{2 \pi} \dot{G}_{\Lambda}\left(\omega_{3}\right) \Gamma_{\Lambda}^{(6)}\left(\omega_{1}, \omega_{2}, \omega_{3}\right) .
\end{aligned}
$$

Using the definition of the scale-dependent anomalous dimension

$$
\eta_{\Lambda}=\Lambda \partial_{\Lambda} \ln Z_{\Lambda},
$$

the flow equation (25b) can be written as

$$
\eta_{\Lambda}=\left.\Lambda Z_{\Lambda} \int \frac{d \omega_{2}}{2 \pi} \dot{G}_{\Lambda}\left(\omega_{2}\right) \frac{\partial \Gamma_{\Lambda}^{(4)}\left(\omega_{1}, \omega_{2}\right)}{\partial\left(i \omega_{1}\right)}\right|_{\omega_{1}=0} .
$$

Because the single-scale propagator $\dot{G}_{\Lambda}(\omega)$ given in Eq. (21) involves a delta function, the frequency integrals in the flow equations (25a), (25c) and in Eq. (27) can easily be evaluated and yield

$$
\begin{aligned}
& \partial_{\Lambda} \Sigma_{\Lambda}(0)=-\frac{Z_{\Lambda}}{\pi} \operatorname{Re}\left[\frac{\Gamma_{\Lambda}^{(4)}(0, \Lambda)}{\mu_{\Lambda}+i \Lambda}\right] \\
& \eta_{\Lambda}=-\frac{\Lambda Z_{\Lambda}^{2}}{\pi} \operatorname{Re}\left[\left.\frac{1}{\mu_{\Lambda}+i \Lambda} \frac{\partial \Gamma_{\Lambda}^{(4)}\left(\omega_{1}, \Lambda\right)}{\partial\left(i \omega_{1}\right)}\right|_{\omega_{1}=0}\right], \\
& \partial_{\Lambda} \Gamma_{\Lambda}^{(4)}\left(\omega_{1}, \omega_{2}\right) \\
&=-J^{2}\left[\dot{\chi}_{\Lambda}\left(\omega_{1}+\omega_{2}\right)+2 \dot{\Pi}_{\Lambda}\left(\omega_{1}-\omega_{2}\right)\right],
\end{aligned}
$$

where we have introduced the flowing chemical potential

$$
\mu_{\Lambda}=Z_{\Lambda}\left(\mu-\Sigma_{\Lambda}(0)\right)
$$


The functions $\dot{\chi}_{\Lambda}(\omega)$ and $\dot{\Pi}_{\Lambda}(\omega)$ are related to the particleparticle and the particle-hole susceptibilities and are given by

$$
\begin{aligned}
\dot{\chi}_{\Lambda}(\omega) \equiv & 2 \int \frac{d \omega_{3}}{2 \pi} \dot{G}_{\Lambda}\left(\omega_{3}\right) G_{\Lambda}\left(\omega-\omega_{3}\right) \\
= & -\frac{Z_{\Lambda}^{2}}{\pi}\left[\frac{1}{\left(\mu_{\Lambda}-i \sigma_{\omega} \Lambda\right)\left(\mu_{\Lambda}+i \omega+i \sigma_{\omega} \Lambda\right)}\right. \\
& \left.+\frac{\Theta(|\omega|-2 \Lambda)}{\left(\mu_{\Lambda}+i \sigma_{\omega} \Lambda\right)\left(\mu_{\Lambda}+i \omega-i \sigma_{\omega} \Lambda\right)}\right] \\
\dot{\Pi}_{\Lambda}(\omega) \equiv & \int \frac{d \omega_{3}}{2 \pi} \dot{G}_{\Lambda}\left(\omega_{3}\right)\left[G_{\Lambda}\left(\omega_{3}-\omega\right)+G_{\Lambda}\left(\omega_{3}+\omega\right)\right] \\
= & -\frac{Z_{\Lambda}^{2}}{\pi} \operatorname{Re}\left\{\frac{1}{\left(\mu_{\Lambda}+i \Lambda\right)\left(\mu_{\Lambda}+i \Lambda+i|\omega|\right)}\right. \\
& \left.+\frac{\Theta(|\omega|-2 \Lambda)}{\left(\mu_{\Lambda}+i \Lambda\right)\left(\mu_{\Lambda}+i \Lambda-i|\omega|\right)}\right\}
\end{aligned}
$$

where $\sigma_{\omega}$ denotes the sign of $\omega$. To simplify the flow equations (28a) and (28c) for the self-energy and the two-body interaction, we neglect the frequency dependence of $\Gamma_{\Lambda}^{(4)}$. This implies that

$$
\begin{aligned}
\Gamma_{\Lambda}^{(4)}(0) & \equiv \Gamma_{\Lambda}^{(4)}\left(\omega_{1}=0, \omega_{2}=0\right) \\
& \approx \Gamma_{\Lambda}^{(4)}\left(\omega_{1}=0, \omega_{2}=\Lambda\right),
\end{aligned}
$$

and leads to the simpler flow equations

$$
\begin{aligned}
& \partial_{\Lambda} \Sigma_{\Lambda}(0)=-\frac{Z_{\Lambda} \Gamma_{\Lambda}^{(4)}(0)}{\pi} \operatorname{Re}\left[\frac{1}{\mu_{\Lambda}+i \Lambda}\right], \\
& \partial_{\Lambda} \Gamma_{\Lambda}^{(4)}(0)=-J^{2}\left[\dot{\chi}_{\Lambda}\left(0^{+}\right)+2 \dot{\Pi}_{\Lambda}(0)\right] .
\end{aligned}
$$

The argument $0^{+}$indicates the limit $\omega \rightarrow 0$ from above, which is necessary because $\sigma_{\omega}$ in $\dot{\chi}_{\Lambda}(\omega)$ is not continuous at $\omega=0$. Note however that the limit from below produces the same result. In summary, we ended up with flow equations for the static part $\Sigma_{\Lambda}(0)$ of the self-energy and the two-body interaction $\Gamma_{\Lambda}^{(4)}(0)$ given in Eqs. (32a) and (32b), respectively. These flow equations depend on the wave function renormalization $Z_{\Lambda}$, which through Eq. (26) is determined by the flowing anomalous dimension given in Eq. (28b). Note that to solve the equation (28b) for the flowing self-energy, the frequency dependence of the four-point vertex cannot be neglected. In order to derive fixed points of the RG flow, we should introduce properly rescaled couplings. Therefore we rescale the fermionic fields as follows:

$$
c_{i}(\omega)=\frac{\sqrt{Z_{\Lambda}}}{\Lambda} \tilde{c}_{i}(\tilde{\omega}),
$$

where $\tilde{\omega}=\omega / \Lambda$ is a dimensionless rescaled frequency. By expressing the effective action in terms of these rescaled fields, the vertices are multiplied by factors of $\sqrt{Z_{\Lambda}}$ and $\Lambda$, which can be absorbed by introducing the rescaled vertices

$$
\tilde{\Gamma}_{\Lambda}^{(2 k)}\left(\tilde{\omega}_{1}, \ldots, \tilde{\omega}_{k}\right)=\frac{Z_{\Lambda}^{k}}{\Lambda} \Gamma_{\Lambda}^{(2 k)}\left(\omega_{1}, \ldots, \omega_{k}\right) .
$$

At this point, it is convenient to parametrize the scale dependence of the couplings in terms of the logarithmic flow parameter

$$
l=\ln \left(\Lambda_{0} / \Lambda\right)
$$

where $\Lambda_{0} \gg J$, and express the self-energy $\Sigma_{\Lambda}(0)$, the vertex $\Gamma_{\Lambda}^{(4)}(0)$, and $Z_{\Lambda}$ in terms of rescaled couplings

$$
\begin{aligned}
& r_{l}=\frac{Z_{\Lambda}^{2}\left(\mu-\Sigma_{\Lambda}(0)\right)^{2}}{\Lambda^{2}}, \\
& u_{l}=\frac{Z_{\Lambda}^{2} \Gamma_{\Lambda}^{(4)}(0)}{\Lambda}, \\
& g_{l}=\frac{J^{2} Z_{\Lambda}^{4}}{\Lambda^{2}} .
\end{aligned}
$$

The coupling $r_{l}$ is the square of the rescaled flowing chemical potential $\tilde{\mu}_{l}=\mu_{\Lambda} / \Lambda$, which is related to the two-point vertex and accordingly has been rescaled as given by Eq. (34). The coupling $u_{l}$ is the rescaled two-body interaction, while $g_{l}$ is introduced for technical reasons to describe the flow of $Z_{\Lambda}$, but also happens to be proportional to the rescaled six-point vertex. This can be seen by noting that according to Eqs. (23) and (34) the six-point vertex $\tilde{\Gamma}_{\Lambda}^{(6)}$ is proportional to $J^{2} Z_{\Lambda}^{3} G_{\Lambda} / \Lambda$ while Eqs. (19) and (24) give

$$
\begin{aligned}
G_{\Lambda}(\omega) & =\frac{\Theta(|\omega|-\Lambda)}{i \omega+\mu-\left[\Sigma_{\Lambda}(0)+\left(1-Z_{\Lambda}^{-1}\right) i \omega\right]} \\
& =\frac{Z_{\Lambda}}{\Lambda} \frac{\Theta(|\tilde{\omega}|-1)}{i \tilde{\omega}+\tilde{\mu}_{\Lambda}} .
\end{aligned}
$$

Using $-\Lambda \partial_{\Lambda}=\partial_{l}$, the flow equations for $Z_{\Lambda}, \Sigma_{\Lambda}(0)$ and $\Gamma_{\Lambda}^{(4)}(0)$ given in Eqs. (26) and (32) can be written in terms of the rescaled couplings as

$$
\begin{aligned}
& \partial_{l} r_{l}=2\left(1-\eta_{l}\right) r_{l}-\frac{2}{\pi} \frac{r_{l} u_{l}}{1+r_{l}}, \\
& \partial_{l} u_{l}=\left(1-2 \eta_{l}\right) u_{l}+\frac{4}{\pi} \frac{g_{l}}{1+r_{l}}\left[\frac{1}{1+r_{l}}-\frac{3}{4}\right], \\
& \partial_{l} g_{l}=2\left(1-2 \eta_{l}\right) g_{l},
\end{aligned}
$$

The scale dependence of the anomalous dimension $\eta_{l}=$ $\eta_{\Lambda}$ is now expressed in terms of $l=\ln \left(\Lambda_{0} / \Lambda\right)$ and is determined by Eq. (28b). In order to close the flow equations, an expression for $\eta_{l}$ is needed. We achieve this by differentiating the flow equation (28c) with respect to the frequency $\omega_{1}$ and formally integrating by $\Lambda$. The result is an integral expression for $\left.\partial_{\omega_{1}} \Gamma_{\Lambda}^{(4)}\left(\omega_{1}, \omega_{2}\right)\right|_{\omega_{1}=0}$ which we insert in Eq. (28b) to obtain an integral equation for the anomalous dimension,

$$
\begin{aligned}
\eta_{l}= & -\frac{g_{l}}{\pi^{2}} \int_{0}^{l} d t e^{2 \int_{l-t}^{l} d \tau \eta_{\tau}} \\
& \times\left\{\operatorname{Re}\left[\frac{1}{\left(i+\tilde{\mu}_{l}\right)\left(-i+\tilde{\mu}_{l-t}\right)\left[i+e^{t}\left(i+\tilde{\mu}_{l-t}\right)\right]^{2}}\right]\right. \\
& \left.-\frac{2}{1+r_{l}} \operatorname{Im}\left[\frac{1}{\left(i+\tilde{\mu}_{l-t}\right)\left[i+e^{t}\left(i+\tilde{\mu}_{l-t}\right)\right]^{2}}\right]\right\} .
\end{aligned}
$$

Analyzing the flow equations (38) we find the fixed points shown in Fig. 1: (i) the unstable vacuum fixed point $V$ at $r=u=g=0$; (ii) the discontinuity fixed point $D$ with one relevant direction; and (iii) the stable NFL fixed point $S_{+}$with $\Sigma(0)=\mu$, preserving scaling. Here, the rescaled two-body interaction $u$ diverges to $+\infty$ and the coupling $g$ approaches 
$\pi^{2} / 3$; and iv) the stable integer-valence fixed point $S_{-}$with $\Sigma(0) \neq \mu$, corresponding to a gapped state yet with a divergent attractive two-body interaction $u \rightarrow-\infty$.

Notice, the large- $N$ self-consistency equations (3) for the self-energy $\Sigma(\omega)$ can be recovered from our FRG approach. This is achieved by using the explicit form (23) of $\Gamma_{\Lambda}^{(6)}$ and applying the Katanin substitution (22) once more on the flow equation (17b) for $\Gamma_{\Lambda}^{(4)}$. The right-hand side of the resulting flow equation can then be written as a total $\Lambda$-derivative so that the flow equation can trivially be integrated to

$$
\begin{aligned}
\Gamma_{\Lambda}^{(4)}\left(\omega_{1}, \omega_{2}\right) \approx & -J^{2}\left[\chi_{\Lambda}\left(\omega_{1}+\omega_{2}\right)\right. \\
& \left.+\Pi_{\Lambda}\left(\omega_{1}-\omega_{2}\right)+\Pi_{\Lambda}\left(\omega_{2}-\omega_{1}\right)\right]
\end{aligned}
$$

with the particle-particle and the particle-hole susceptibilities

$$
\begin{aligned}
& \chi_{\Lambda}(\omega)=\frac{1}{\beta} \sum_{\omega_{3}} G_{\Lambda}\left(\omega_{3}\right) G_{\Lambda}\left(\omega-\omega_{3}\right), \\
& \Pi_{\Lambda}(\omega)=\frac{1}{\beta} \sum_{\omega_{3}} G_{\Lambda}\left(\omega_{3}\right) G_{\Lambda}\left(\omega_{3}-\omega\right) .
\end{aligned}
$$

Substituting the four-point vertex (40) into the flow equation (17a) for the self-energy, applying a third Kataninsubstitution, and integrating over the flow parameter $\Lambda$ yields

$$
\Sigma(\omega)=-\frac{J^{2}}{\beta^{2}} \sum_{\omega_{1} \omega_{2}} G\left(\omega_{1}\right) G\left(\omega_{2}\right) G\left(\omega_{1}+\omega_{2}-\omega\right),
$$

which is equivalent to Eq. (3).

In order to characterize the fermionic dynamics we investigate the scale-dependent anomalous dimension $\eta_{l}$. Therefore we integrate the flow equations (38) for $r_{l}, u_{l}$ and $g_{l}$ together with the integral equation (39) for $\eta_{l}$ numerically with slightly varying initial conditions near the vacuum fixed point to obtain the trajectories shown in Fig. 1. The corresponding flows of the anomalous dimension are shown in the top panel of Fig. 6. As expected, the NFL fixed point $S_{+}$has $\eta=1 / 2$ in agreement with Eq. (5), while we find $\eta=0$ for the integervalence fixed point $S_{-}$, consistent with Eq. (8). The most remarkable finding of our analysis is however that $\eta=1 / 2$ at the discontinuity fixed point, see lower part of Fig. 6. This can be shown analytically as will be explained in Sec. IV. While this fixed point describes a discontinuous change of the particle number, the fermionic dynamics is quantum critical. The first-order transition is characterized by critical slowing down of the fermions.

\section{DISCONTINUITY FIXED POINT}

\section{A. Scaling close to the discontinuity fixed point}

At the discontinuity fixed point $D$, all rescaled couplings approach finite limits. This enables us to analyze the linearized flow in its vicinity. Using $\eta=1 / 2$, determined earlier, we can determine the numerical values of our couplings by demanding that all scale derivatives in Eq. (38) vanish. We obtain

$$
r_{*}=\frac{1}{3}, \quad u_{*}=\frac{2 \pi}{3}, \quad g_{*}=\frac{1}{2 c} \approx 8.52 .
$$

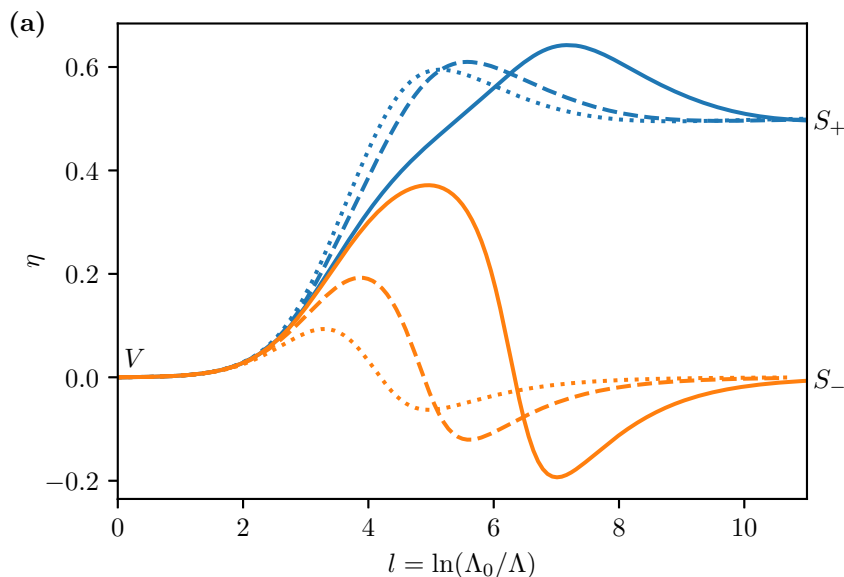

(b)

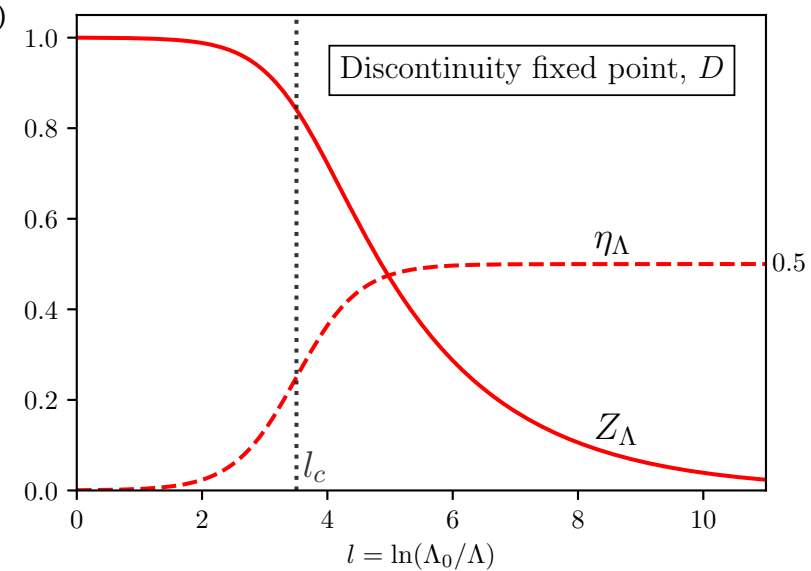

FIG. 6. (a) RG flow of the anomalous dimension for different initial conditions near the vacuum fixed point. The NFL sink $S_{+}$yields $\eta=1 / 2$ (blue curves), while the orange curves flow into the integervalence fixed point $S_{-}$where the system is gapped and $\eta=0$. The colors and styles of the lines correspond to the trajectories in Fig. 1. (b) Flow of the wave-function renormalization $Z_{\Lambda}$ and the scaledependent anomalous dimension $\eta$ right at the discontinuity fixed point $D$. The dashed line marks the crossover scale $l_{c}=\ln \left(\Lambda_{0} / \Lambda_{c}\right)$ determined by Eq. (49).

The result for $g_{*}$ follows from an analysis of Eq. (39) near $D$ which yields $\eta_{\Lambda}=c g_{\Lambda}$ with numerical coefficient $c=$ $3\left(\ln 2-\frac{1}{2}\right) / \pi^{2} \approx 0.0587$. The precise value of $c$ may weakly depend on details of the cutoff scheme. From Fig. 1, we see that the discontinuity fixed point has an unstable and a stable direction. To calculate the corresponding scaling variables, we linearize the flow in its vicinity. Setting $r_{l}=r_{*}+\delta r_{l}$, and similar for $u_{l}$ and $g_{l}$, we obtain the linearized flow equations. Within our truncation $\delta g_{l}$ decouples and we can focus on the $\delta r-\delta u$ plane. With $\delta u_{l}=\frac{3}{4} \sqrt{\frac{3}{c}} \delta y_{l}$, we obtain

$$
\partial_{l}\left(\begin{array}{l}
\delta r_{l} \\
\delta y_{l}
\end{array}\right)=\left(\begin{array}{cc}
\frac{1}{4} & -a \\
-a & 0
\end{array}\right)\left(\begin{array}{l}
\delta r_{l} \\
\delta y_{l}
\end{array}\right),
$$

with numerical constant $a=\frac{3}{8 \pi} \sqrt{3 / c}$. The eigenvalues of the matrix are $\lambda_{+} \approx 0.987$ and $\lambda_{-}=1 / 4-\lambda_{+}$. The corresponding eigen-directions in the $r$ - $u$ plane follow easily. Near the transition, the relevant eigenvector is proportional to $\mu-\mu_{*}$. Hence, near the transition, the grand canonical potential per 
fermion species should - in addition to a regular piece-be characterized by a singular contribution with scaling behavior,

$$
\Omega(\mu, T)=\Omega_{\mathrm{reg}}(\mu, T)+e^{-l} \Omega_{\text {sing }}\left(e^{\lambda_{+} l}\left(\mu-\mu_{*}\right), e^{l} T\right) .
$$

This yields for the particle density

$$
n=-\partial \Omega / \partial \mu=n_{\text {reg }} \pm B_{ \pm}\left|\mu-\mu_{*}\right|^{-1+1 / \lambda_{+}} .
$$

Keeping in mind that our determination of $c$ is approximate, the above result for $\lambda_{+}$is consistent with $\lambda_{+}=1$, yielding indeed a discontinuity of $n$ at $\mu_{*}$. According to scaling arguments near first-order transitions $[55,56]$ the relevant eigenvalue is the space dimension, i.e., $\lambda_{+}=d$. For our zerodimensional quantum system with one time direction, this yields indeed $\lambda_{+}=1$; see also Appendix A.

The scaling dimension of the grand canonical potential, i.e., the coefficient $e^{-l}$ in front of $\Omega_{\text {sing }}$, further ensures that the entropy $S=-\partial \Omega / \partial T$ jumps a $T=0$ from its finite value in the NFL phase to zero in the integer-valence phase. The jump in the zero-point entropy is visible in Fig. 2 through the low- $T$ slope of the transition temperature together with the Clausius-Clapeyron relation $d T_{c} / d \mu=-\Delta n / \Delta S$.

The linear relation between $\eta_{\Lambda}$ and $g_{\Lambda}$ near the discontinuity fixed point can also be used to determine the crossover energy to the regime with $\eta \approx \frac{1}{2}$. Expressing $g_{\Lambda}$ and $\eta_{\Lambda}$ in terms of $Z_{\Lambda}$, we obtain the closed flow equation of the wavefunction renormalization near $D$,

$$
\partial_{\Lambda} Z_{\Lambda}=c J^{2} \Lambda^{-3} Z_{\Lambda}^{5}
$$

The solution of this differential equation with initial condition $Z_{\Lambda_{0}}=1$ is

$$
Z_{\Lambda}=\frac{1}{\left[1+\Lambda_{c}^{2}\left(\frac{1}{\Lambda^{2}}-\frac{1}{\Lambda_{0}^{2}}\right)\right]^{1 / 4}}=\frac{\sqrt{\frac{\Lambda}{\Lambda_{c}}}}{\left[1+\Lambda^{2}\left(\frac{1}{\Lambda_{c}^{2}}-\frac{1}{\Lambda_{0}^{2}}\right)\right]^{1 / 4}},
$$

where the crossover energy scale $\Lambda_{c}$ is given by

$$
\Lambda_{c}=\sqrt{2 c} J=\frac{\sqrt{6 \ln 2-3}}{\pi} J \approx 0.343 J .
$$

The scale-dependent anomalous dimension $\eta_{\Lambda}$ for a RG trajectory flowing into the discontinuity fixed point is

$$
\eta_{l}=\frac{\Lambda \partial_{\Lambda} Z_{\Lambda}}{Z_{\Lambda}}=\frac{\Lambda_{c}^{2}}{2} \frac{Z_{\Lambda}^{4}}{\Lambda^{2}}=\frac{\frac{1}{2}}{1+\Lambda^{2}\left(\frac{1}{\Lambda_{c}^{2}}-\frac{1}{\Lambda_{0}^{2}}\right)},
$$

which approaches $1 / 2$ for $\Lambda \rightarrow 0$. The crossover scale $\Lambda_{c}=$ $\sqrt{2 c} J \approx 0.343 J$ is marked by the dotted line in the lower part of Fig. 6.

\section{B. The physical meaning of the discontinuity fixed point}

Let us point out an aspect of our analysis that is crucial to put it into context: the quantum phase transition of the SYK model as a function of chemical potential is a genuine, discontinuous first-order phase transition. In the thermodynamic limit the system is either in one or in the other phase. This view is consistent with the fact that ultimately, one of the two stable fixed points $S_{ \pm}$governs the behavior of the system. Indeed, both fixed points describe vastly distinct physical regimes, a non-Fermi liquid or a state of integer valence. Hence, the power-law behavior of the fermionic self-energy at the discontinuity fixed point, reminiscent of critical behavior, seems at odds with the discontinuous character of the transition. The apparent contradiction can be resolved if one realizes that this power law describes the flow towards the discontinuity fixed point. In the context of classical first-order transitions, the issue of scaling at a discontinuous transition has been addressed by Fisher and Berker [56]. They showed that, despite the discontinuous nature of the transition, one can develop a scaling approach, where the first-order transition can be understood as a new fixed point. The correct scaling exponent of the tuning parameter, in our case the chemical potential, then ensures a discontinuity of thermodynamic quantities in the thermodynamic limit.

In addition to these established aspects of first-order transitions, we find a fermionic anomalous dimension of $\eta=1 / 2$ at the discontinuity fixed point. Although this coincides with the anomalous dimension in the non-Fermi liquid phase, the physical behavior is distinct as we show in Sec. IV C. This leads us to the conclusion that the SYK model displays a behavior that has, to our knowledge, not been observed before: the fermionic self-energy at the discontinuity fixed point shows power-law behavior, which indicates a critical state of matter.

The question arises whether this could be a manifestation of new physics near or at the quantum first-order transition. We are not able to give a definite answer to this question, but to gain some more insight we present in Appendix A a detailed analysis of the scaling behavior near a quantum first-order transition in quantum rotor and transverse field Ising models. There, the flow towards the discontinuity fixed point can be viewed as the behavior of an increasingly large, but finite system. While the transition is discontinuous in the limit $N \rightarrow \infty$, it is softened in finite systems. The quantum mechanical state in the transition region is then an entangled superposition of the two phases and has a characteristic persistence timescale $\tau_{p}$ (inverse tunneling rate) that diverges as the transition becomes sharp. This timescale is the analog to the corresponding persistence length that was discussed for classical discontinuity fixed points [56]. Hence, the flow towards the discontinuity fixed point and the power-law behavior of the self-energy are only relevant if one softens the transition.

For the SYK model, finite- $N$ corrections are subtle [61] and we do not know the precise fate of the first-order transition. The analysis in Appendix A is nevertheless useful for our problem, as any perturbation that softens the transition will give rise to scaling behavior governed by the discontinuity fixed point. An example was presented in Ref. [35], where a generalization of the SYK model to a lattice system was introduced; in this model, the discontinuity is softened but a rapid change in the valence remains visible, i.e., the behavior of the system should be governed by the discontinuity fixed point.

We do not know whether an entangled quantum state in the softened transition region of the SYK model persists in the limit where the transition becomes sharp. However, in view of our result for the fermionic anomalous dimension at the discontinuity fixed point, we suspect a connection between an entangled state at the transition and critical dynamics of 
the fermions. To test this hypothesis of critical coexistence, it would be desirable to analyze generalizations of the SYK model exhibiting different anomalous exponents. The finding of variable exponents in related Yukawa-SYK models [48], that even change as a function of density [36], is encouraging.

A related question concerns a possible holographic dual of the coexistence state. In Ref. [37], the first-order phase transition in the SYK model is identified with the HawkingPage transition between an Anti-de-Sitter (AdS) space-time with a black hole and an AdS space-time without black hole. Naively, an entangled state at phase coexistence would imply a superposition of space-time metrics, which is not compatible with general relativity. In fact, the authors of Ref. [37] state that general relativity alone is not sufficient to establish the duality between SYK model and AdS space-time. It would therefore be interesting to examine the concept of critical coexistence in the context of the holographic duality.

\section{How to distinguish the non-Fermi liquid phase from the critical coexistence phase}

Since the non-Fermi liquid fixed point $S_{+}$and the discontinuity fixed point $D$ are characterized by the same anomalous dimension $\eta=1 / 2$, the question arises of how to distinguish the phases of matter associated with these fixed points in practice. We now show that the behavior of the effective gap

$$
\Delta_{\Lambda}=\mu-\Sigma_{\Lambda}(0)
$$

at some small cutoff scale $\Lambda \ll J$ can be used to distinguish these two different phases of matter. Within the framework of the FRG this follows from the fact that at $D$ the rescaled coupling

$$
r_{l}=\frac{Z_{\Lambda}^{2}\left(\mu-\Sigma_{\Lambda}(0)\right)^{2}}{\Lambda^{2}}=\frac{Z_{\Lambda}^{2} \Delta_{\Lambda}^{2}}{\Lambda^{2}}
$$

introduced in Eq. (36a) approaches a finite limit $r_{*}>0$, whereas $\lim _{l \rightarrow \infty} r_{l}$ vanishes if we approach the non-Fermi liquid fixed point. This implies very different behavior of the effective gap $\Delta_{\Lambda}$ for finite $\Lambda$. To see this, let us explicitly solve the RG flow equations (38) in the vicinity of the NFL fixed point and the discontinuity fixed point. Note that in both cases the effective gap $\Delta_{\Lambda}$ vanishes for $\Lambda \rightarrow 0$, but for finite $\Lambda \ll J$ the gap for an RG trajectory flowing into $S_{+}$is much smaller than the gap for a trajectory flowing into $D$, as we show now.

Consider first an RG trajectory which flows into the discontinuity fixed point. Given the fact that according to Eq. (43) the rescaled coupling $r_{l}$ approaches in this case the limit $r_{*}=1 / 3$ and that $Z_{\Lambda} \sim \sqrt{\Lambda / \Lambda_{c}}$, we conclude from (52) that the scaling of $\Delta_{\Lambda}$ for small $\Lambda$ is determined by

$$
\left.\Delta_{\Lambda} \approx \sqrt{r_{*} \Lambda_{c} \Lambda} \propto \sqrt{J \Lambda} \quad \text { (gap close to } D\right) .
$$

Next, consider an RG trajectory which flows into the NFL fixed point $S_{+}$where $r_{*}=0$ and $g_{*}$ is a finite positive constant. By inspection of the RG trajectories flowing into $S_{+}$shown in Fig. 1, it is already obvious that the couplings $r_{l}$ and $g_{l}$ approach their fixed point values much faster than the interaction $u_{l}$. As a consequence, the RG flow close to $S_{+}$can be obtained by replacing $r_{l} \rightarrow 0, g_{l} \rightarrow g_{*}$ and $\eta_{l} \rightarrow 1 / 2$ on the right-hand side of the flow equations (38), which then reduce to

$$
\begin{aligned}
\partial_{l} \ln r_{l} & =1-\frac{2}{\pi} u_{l}, \\
\partial_{l} u_{l} & =\frac{g_{*}}{\pi} .
\end{aligned}
$$

The solution for $r_{l}$ with initial condition $r_{l=l_{1}}=r_{1}$ and $u_{l=l_{1}}=u_{1} \ll 1$ is

$$
r_{l}=r_{1} \frac{\Lambda_{1}}{\Lambda} e^{-\frac{g_{*} *}{\pi^{2}} \ln ^{2}\left(\Lambda_{1} / \Lambda\right)},
$$

where $\Lambda_{1}=\Lambda_{0} e^{-l_{1}}$ is the scale where the flowing $\eta_{l}$ approaches its fixed-point value of $1 / 2$. With $\Lambda_{1}=\mathcal{O}(J)$ and $r_{1}=\mathcal{O}(1)$, we finally obtain

$$
\Delta_{\Lambda} \propto J e^{-\frac{g *}{\pi^{2}} \ln ^{2}(J / \Lambda)} \quad\left(\text { gap close to } S_{+}\right),
$$

which for a given $\Lambda \ll J$ is much smaller than the effective gap close to the discontinuity fixed point in Eq. (53). A more accurate direct numerical solution of the flow equations (38) confirms our simplified analysis.

The above results suggest that the phases of matter associated with $S_{+}$and $D$ can be distinguished by adding a small but relevant perturbation (for example, a random chemical potential or some hopping destroying the zero dimensionality) which destabilizes both fixed points $S_{+}$and $D$. As long as the associated energy scale $U$ is small compared with $\mathrm{RG}$ cutoff scale $\Lambda$, we expect that the RG trajectories are only weakly perturbed, but at scale $\Lambda \approx U$, the RG flow will start to flow away from the fixed points. By measuring the effective gap $\Delta_{\Lambda=U}$ of the fermionic spectrum we can in principle distinguish the non-Fermi liquid phase associated with the fixed point $S_{+}$from the critical coexistence phase associated with the discontinuity fixed point $D$. The effective gap can be determined from the low-frequency behavior of the spectral function. Consider first the NFL phase, where the large- $N$ solution of the self-energy is $\Sigma_{\mathrm{NFL}}(\omega)=\mu-C_{\omega}|\omega|^{1 / 2}$ and the coefficient $C_{\omega}$ only determines the signs of the real and imaginary parts, see Eqs. (5) and (6). This implies $\Sigma_{\mathrm{NFL}}(0)=$ $\mu$, which determines the low-frequency spectral function

$$
A_{\mathrm{NFL}}(\omega)=-\frac{1}{\pi} \operatorname{Im} G_{\mathrm{NFL}}^{\mathrm{ret}}(\omega) \propto|\omega|^{-1 / 2},
$$

where the retarded Green function $G_{\mathrm{NFL}}^{\mathrm{ret}}(\omega)$ is obtained from its Matsubara counterpart $G_{\mathrm{NFL}}(\omega)$ via analytic continuation $i \omega \rightarrow \omega+i 0^{+}$. Let us instead assume that $\Delta \neq 0$ is a fixed constant, while the frequency dependence of the self-energy continues to be governed by $\eta=1 / 2$. Then follows at low frequencies $|\omega| \ll \Delta$ for the spectral function

$$
A(\omega) \propto \frac{|\omega|^{1 / 2}}{\Delta^{2}},
$$

which is qualitatively distinct from Eq. (57). The situation close to the discontinuity fixed point $D$ is more subtle, as asymptotically $\Delta_{\Lambda \rightarrow 0} \rightarrow 0$. However, in distinction to the NFL flow Eq. (56), where at $\Delta_{\Lambda} \ll \Lambda$ at small $\Lambda$, it follows from (53) that $\Delta_{\Lambda} \gg \Lambda$ close to $D$ if $\Lambda \ll r_{*} \Lambda_{c}$. Since $r_{*}$ is of order unity it also holds that $\eta_{\Lambda} \approx 1 / 2$ in this regime. An actual analysis of the spectral function within the FRG requires to go beyond the analysis presented here. We do, 


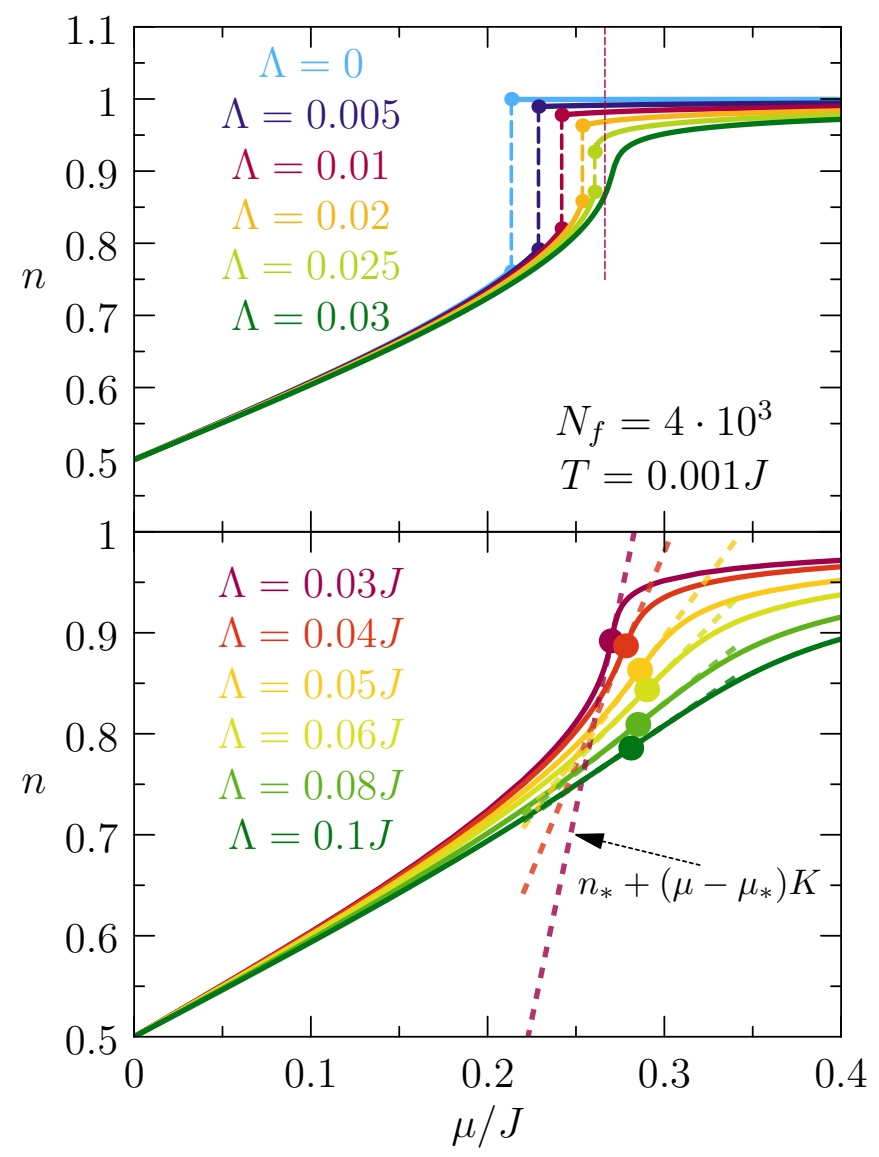

FIG. 7. Fermion density $n$ as a function of chemical potential $\mu$ at different $\Lambda$. In the bottom panel, $n_{*}\left(\mu_{*}\right)$ denotes the inflection point, which substitutes for the density discontinuity when the firstorder transition is smeared out. The dashed lines show the linear expansion of $n(\mu)$ at the inflection point (dots) with slope $K$.

however, expect that the distinct scaling behavior of $\Delta_{\Lambda}$ will have significant impact on the spectral function close to the discontinuity fixed point if compared to the two other phases.

To strengthen the above argument and to connect our FRG results with the large- $N$ analysis, we have also considered the saddle-point equations on the imaginary axis with a finite infrared cutoff $\Lambda$,

$$
\begin{aligned}
& G_{\Lambda}(\omega)=\frac{\Theta(|\omega|-\Lambda)}{i \omega+\mu-\Sigma_{\Lambda}(\omega)}, \\
& \Sigma_{\Lambda}(\tau)=-J^{2} G_{\Lambda}^{2}(\tau) G_{\Lambda}(-\tau) .
\end{aligned}
$$

Using the methods outlined in Appendix C, we have solved Eqs. (59) self-consistently for $G_{\Lambda}(\omega)$ and $\Sigma_{\Lambda}(\omega)$. The original model (3), which produces the phase diagram in Fig. 2, is recovered in the limit $\Lambda \rightarrow 0$. By numerically computing the fermion density $n(\mu)$ for fixed $\Lambda$ at $T=0.001 J$ (close to zero temperature), we find that for $\Lambda>\Lambda_{t} \approx 0.025 \mathrm{~J}$ the density discontinuity at the first-order phase transition is smeared out into a continuous $n(\mu)$ curve; more precisely, the discontinuity in $n(\mu)$ occurring for $\Lambda<\Lambda_{t}$ changes into a flex point $n_{*}\left(\mu_{*}\right)$ for $\Lambda>\Lambda_{t}$ as shown in Fig. 7 .

Our numerical results for the effective gap $\Delta_{\Lambda}$ defined in Eq. (51) confirm the results of our RG analysis: the depen-

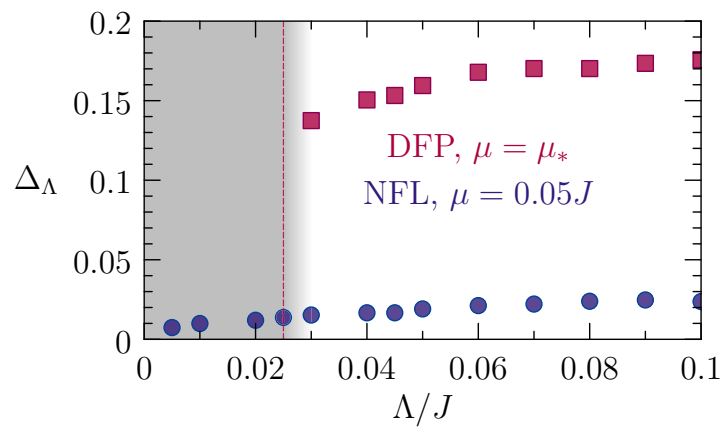

FIG. 8. Scale-dependent gap $\Delta_{\Lambda}=\mu-\Sigma_{\Lambda}(0)$ between $\mu$ and the extrapolated zero-frequency self-energy $\Sigma_{\Lambda}(0)$, as a function of cutoff $\Lambda$.

dence of the effective gap $\Delta_{\Lambda}$ on $\Lambda$ is qualitatively different for the non-Fermi liquid phase and the coexistence phase associated with the discontinuity fixed point. This difference is evident in Fig. 8, where the blue circles show the calculations deep in the NFL phase at $\mu=0.05 \mathrm{~J}$, while the red squares mark the results at the coexistence point $\mu=\mu_{*}$. The gray region corresponds to $\Lambda<\Lambda_{t}$ where a finite density discontinuity persists at the first-order transition. The finite-temperature numerics suggest $\Delta_{\Lambda} \propto \Lambda^{\alpha}$ with $\alpha \gg 1$ in the non-Fermi liquid phase, which is consistent with our RG result (56). On the other hand, at the coexistence point we obtain $\Delta_{\Lambda} \propto \Lambda^{\alpha}$ with $0<\alpha<1$. Given the limited range of $\Lambda$ used for the fit, this is consistent with Eq. (53).

\section{INTEGER-VALENCE FIXED POINT}

Finally, we elucidate our finding that the rescaled twobody interaction $u_{l}$ at the integer valence fixed point $S_{-}$, shown in Fig. 1, flows to $-\infty$, which corresponds to a finite attractive unrescaled two-body interaction. Of course, the single-particle properties for occupations $n=0$ or 1 are trivial. A single electron or hole added to the system has no particle to interact with. This does not apply to higher-particle correlations. Following the classical work of Galitskii [62], the two-particle vertex of a such a dilute system can be obtained by summing up all particle-particle ladder diagrams. We perform this analysis prior to the disorder averaging,

$$
\Gamma_{i j k l}(\omega)=J_{i j k l}-\sum_{m<n} J_{i j m n} \Gamma_{m n k l}(\omega) \chi(\omega),
$$

with particle-particle bubble $\chi(\tau)=G^{2}(\tau)$. Summing up the series and averaging term-by-term over the $J_{i j k l}$ yields at large $N$ for $i<j$ and $k<l$ and after analytic continuation to retarded functions,

$$
\Gamma_{i j k l}^{\mathrm{ret}}(\epsilon-2 \mu)=-\delta_{i k} \delta_{j l} \epsilon\left(1-\frac{2}{1+\sqrt{\frac{\epsilon^{2}-4 J^{2} / N}{\epsilon^{2}}}}\right) .
$$

For details on the derivation of this result, see Appendix C. A plot of the real and imaginary part of this vertex is shown in Fig. 9. The imaginary part

$$
\operatorname{Im} \Gamma_{i j i j}^{\mathrm{ret}}(\omega)=-\frac{(\omega+2 \mu)^{2}}{2 J^{2} / N} \sqrt{4 J^{2} / N-(\omega+2 \mu)^{2}}
$$




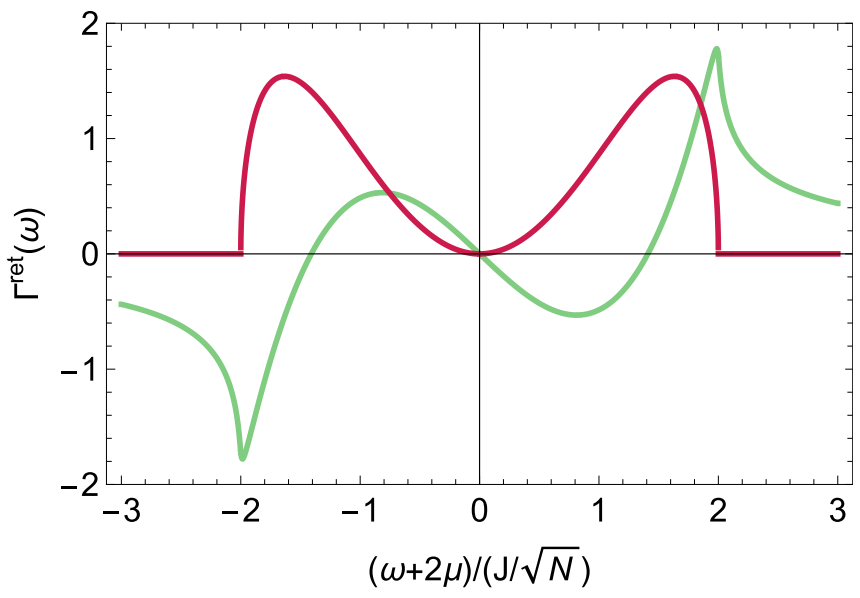

FIG. 9. Real part (green) and -1 times the imaginary part (red) of the two-particle scattering vertex $\Gamma_{i j i j}^{\mathrm{ret}}$ as a function of frequency. A finite imaginary part signals scattering states.

is finite in the interval $-2 \mu \mp 2 J / \sqrt{N}$. The vertex vanishes right at the trivial two-particle energy $-2 \mu$. However, a band of two-particle scattering states around the energy $-2 \mu$ with band width $4 J / \sqrt{N}$ emerges. Injecting two particles with different flavors enables them to scatter into each other and a band of bonding and antibonding states occurs. While the single-particle physics of the integer-valence phase is trivial and individual electrons or holes are completely immobile, pairs of correlated particles propagate coherently, a behavior that is signaled by the flow of the coupling constant $u \rightarrow-\infty$ shown in Fig. 1.

\section{CONCLUSIONS AND OUTLOOK}

In summary, we formulated the functional RG of the SYK model and determined its global renormalization group flow. Our approach reproduces known results of the two stable phases, the non-Fermi liquid state and the integer-valence state, see Fig. 2. The crucial insight of our manuscript is that two known phases are separated by a first-order transition with thus far unknown critical dynamics at coexistence. Such criticality at coexistence is a result that has, to our knowledge, not been obtained in the description of first-order transitions in correlated fermion systems. It is a unique and surprising quantum effect and possibly rooted in quantum mechanical entanglement of the two states that are separated by the firstorder transition. Fermionic excitations at phase coexistence are characterized by an anomalous dimension and behave quantum critical. This behavior cannot be captured in terms of a local Ising variable, a concept that was highly successful for classical symmetry-preserving first-order transitions.

The fact that one can perform a controlled analytic expansion in the vicinity of this discontinuity fixed point illustrates the power of FRG to describe strong-coupling states of matter. The relevant scaling dimension of the discontinuity fixed point is found to be consistent with the behavior near first-order transitions [55,56]. This rules out an interpretation that the discontinuity fixed point describes one of the two spinodal points where the globally unstable solution also becomes locally unstable.
To further connect our results to Refs. [34,37], it would be interesting to generalize our FRG procedure to finite temperatures and to examine the scaling behavior at the critical endpoint of the first-order transition. A generalization to more complicated models like the Yukawa-SYK model as discussed in Ref. [36] or coupled SYK models as discussed in Refs. $[63,64]$ may be useful to better understand the phase transition and the duality to black hole physics.

Second-order quantum phase transitions have captivated the community of correlated quantum systems for several decades by now. In contrast, first-order quantum phase transitions, albeit more abundant in nature, have received much less attention. A frequent belief is that no genuine universal insight can be gained here. One is either in one phase or in the other; the distinction is based on the energetics of largely dissimilar states. Our results reveal that a quantum first-order transition that does not break a symmetry can be dramatically altered by the presence of universal non-Fermi-liquid electronic excitations.

\section{ACKNOWLEDGMENTS}

This work was funded by the Deutsche Forschungsgemeinschaft (DFG, German Research Foundation) - TRR 288 422213477 (project A07). We are grateful to M. A. Axenovich for pointing out the role of Catalan numbers in the disorder average and to M. Garst for helpful discussions.

\section{APPENDIX A: DISCONTINUITY FIXED POINT IN QUANTUM SYSTEMS}

In this Appendix, we summarize the scaling behavior of quantum first-order transitions, i.e., the behavior in the vicinity of a discontinuity fixed point (DFP) at or near $T=0$. Scaling near a DFP was initially formulated for classical firstorder transitions $[55,56]$. The generalization to the quantum regime, that we discuss for several model systems, will help us with the interpretation of the results obtained in the main part of the manuscript. The first-order transition discussed in this paper is similar to the original liquid-vapor transition in that the two phases are not related by a symmetry operation. The finite-size scaling for first-order transitions was performed in Ref. [65]. As was discussed in Ref. [66] the scaling near an asymmetric transition can be directly related to one where the two phases are connected by a symmetry. Hence, we focus our attention here only to this simplified scenario.

\section{Finite-size scaling at first-order phase transitions}

Let $h$ be the external variable that tunes the system through the transition, located at $h=0$. In our problem of the SYK model, $h=\mu-\mu_{*}$. Then the singular piece of the free energy density is assumed to obey the scaling relation

$$
f(h)=b^{-d} f\left(b^{\lambda} h\right) .
$$

In what follows, we will only consider this singular piece and will use the letter $f$ for the free energy density rather than $\Omega$, used in the main part of the paper for the grand canonical potential that occurs when one tunes the transition with a chemical potential. For the order parameter $\phi=-\partial f / \partial h$ 
follows the scaling form

$$
\phi(h)=b^{\lambda-d} \phi\left(b^{\lambda} h\right) .
$$

This scaling form is formally identical to the well-established one near classical second-order transitions and critical points, were $\lambda=d \delta /(1+\delta)$ with critical exponent $\delta$ that describes $\phi \sim h^{1 / \delta}$. At the discontinuity fixed point, the value of $\lambda$ is not determined by critical fluctuations. Instead, it ensures the expected discontinuous change of the order parameter. Setting $b^{\lambda} h= \pm 1$ depending on the sign of $h$, it follows

$$
\phi(h)=|h|^{\frac{\lambda-d}{\lambda}} \phi(\operatorname{sign}(h)) .
$$

Obviously, the only way this is consistent with a discontinuous order parameter is $\lambda=d$. Then $\phi$ jumps by $\Delta \phi=\phi(1)-$ $\phi(-1)$. Thus, while Eq. (A1) is formally the same as for critical points, the special value of the scaling dimension $\lambda$ yields very different behavior. It is nevertheless tempting to associate the scaling variable $b=h^{1 / d}$ with some underlying length scale. In Ref. [56], it was discussed that $\xi_{p} \sim h^{-1 / d}$ should not be confused with the usual correlation length. Instead, it corresponds to a persistence length for the coexisting phases. Its divergence corresponds to long-range order in correlation functions that distinguish the two phases [56]. One can even analyze scaling laws such as $2-\eta=d(\delta-1) /(\delta+1)$, use $\delta=\infty$ that follows from $\lambda=d$ and obtain $\eta=2-d$, which corresponds to no decay of the usual correlation functions.

Overall, this scaling approach seems to primarily describe what is known already, namely that the order parameter at a first-order transition is discontinuous. The real power of the approach becomes evident when one analyses finite-size scaling near a transition $[55,56,67,68]$. To have a uniform notation that also applies to our subsequent discussion of fully connected models, we use instead of the linear size $L$ the total size $N=L^{d}$. For the scaling law follows now

$$
f(h, N)=b^{-d} f\left(b^{d} h, b^{-d} N\right) .
$$

The dimension for the system size follows from the usual scaling of the linear dimension $L \rightarrow b^{-1} L$. For the order parameter follows with the choice $b^{d}=N$ for the flow variable $b$ that $\phi(h, N)=\phi(h N, 1)$. Since the susceptibility of a finite system should be finite, it must hold that for small $|h| N$ the function $\phi(h N, 1)$ is linear in its argument,

$$
\phi(h, N) \propto h N .
$$

While the order parameter of a finite system vanishes at $h=0$, its slope increases with $N$ and eventually leads to the discontinuous jump for $N \rightarrow \infty$. The susceptibility $\chi=$ $\partial \phi /\left.\partial h\right|_{h \rightarrow \pm 0} \propto N$ grows linear with the system size and eventually becomes the $\delta(h)$ at infinite $N$. The same happens for the heat capacity and the integral over the $\delta$-function yields the latent heat.

The reason why the scaling approach offers insights into the finite-size behavior is that a finite $N$ washes out the sharp phase transition. More generally, any external variable that accomplishes the same can be efficiently characterized using these scaling arguments. An interesting example are firstorder line transitions [69] that occur at the one-dimensional edge of a two-dimensional system and are an example where genuine critical behavior and first-order scaling behavior occur simultaneously.

\section{Finite-size scaling at discontinuous quantum phase transitions}

Let us next discuss the behavior near quantum first-order transitions. Scaling at quantum first-order transitions was, for example, discussed in Refs. [70-73]. In particular, critical behavior at a quantum discontinuity fixed point was discussed in the context of a real-space renormalization group analysis of Potts models [70,71]. The finite-size scaling analysis presented here follows closely Refs. [74,75]. The scaling theory of quantum systems can be generalized to

$$
f(h, T, N)=b^{-d-z} f\left(b^{d+z} h, b^{z} T, b^{-d} N\right),
$$

where the crucial new ingredient is the dynamical scaling dimension $z$. We will see that the dynamics underlying this scaling dimension is quite different compared to the one at a second-order transition. Following a logic similar to the one for the classical case, Eq. (A6) yields at $T=0$ for the order parameter $\phi(h, N)=\phi\left(b^{d+z} h, b^{-d} N\right)=\phi\left(h N^{1+z / d}, 1\right)$. Under the assumption of a finite susceptibility at finite $N$ follows for small field,

$$
\phi(h, N) \propto h N^{1+\frac{z}{d}} .
$$

Below we will also discuss a case where the assumption of a finite susceptibility does not hold. For now, we assume that this is the case. The key observation is that the order parameter grows much faster with $N$ than near a classical transition. We will see that this additional enhancement is a consequence of the soft excitation gap

$$
\Delta \sim N^{-z / d}
$$

that governs fluctuations in the time directions and enhances the order-parameter susceptibility

$$
\chi \sim \frac{N}{\Delta}
$$

through quantum transitions of the entire finite system.

\section{Finite range quantum rotor model}

We start our analysis of specific solvable models with a finite-range system and consider an $M$-component quantum rotor problem in the large- $M$ limit. Below we will see that the limitation to large values of $M$ is not an important restriction. We start from the action of an $M$-component vector field $\boldsymbol{\phi}(x)=\left(\phi_{1}(x), \cdots, \phi_{M}(x)\right)$,

$$
S=\int_{x, y} \phi(x) G_{0}^{-1}(x-y) \cdot \phi(y)+\frac{u}{2 M} \int_{x}(\phi(x) \cdot \phi(x))^{2},
$$

where $x=(\boldsymbol{x}, \tau)$ comprises position $\boldsymbol{x}$ and imaginary time $\tau$, and $\int_{x} \cdots=\int d^{d} \boldsymbol{x} \int_{0}^{\beta} d \tau \cdots$. For the Fourier transform of the bare propagator we use

$$
G_{0}^{-1}\left(\boldsymbol{q}, \omega_{n}\right)=r_{0}+\boldsymbol{q}^{2}+\gamma\left|\omega_{n}\right|^{\alpha},
$$

where we treat the exponent $\alpha$ as input parameter. The value $\alpha=2$ corresponds to a problem with a ballistic spectrum [76], while $\alpha=1$ has been discussed in the context of itinerant 
electron problems with particle-hole excitations [77-79]. An arbitrary exponent $\alpha$ can also be generated via coupling $\phi(x)$ to an external bath of harmonic oscillators, see for example [80]. Then $\alpha=1$ corresponds to an Ohmic bath. $\alpha>1$ is referred to a super-Ohmic and $\alpha<1$ to a sub-Ohmic system, respectively. We consider a finite system of size $N=L^{d}$. The large- $M$ self-consistency equations are then given as [74]

$$
\begin{aligned}
& r=r_{0}+u\left\langle\phi^{2}\right\rangle+u \phi_{0}^{2}, \\
& h=r \phi_{0},
\end{aligned}
$$

where $\phi_{0}=\left\langle\boldsymbol{\phi} \cdot \boldsymbol{e}_{h}\right\rangle / \sqrt{M}$ is the appropriately normalized order parameter and

$$
\left\langle\phi^{2}\right\rangle=\frac{T}{N} \sum_{\boldsymbol{q} \omega_{n}} \frac{1}{r+\boldsymbol{q}^{2}+\gamma\left|\omega_{n}\right|^{\alpha}}
$$

describes the self-consistently determined order-parameter fluctuations. We included a conjugate field $\boldsymbol{h}=\boldsymbol{h}_{h}$ that determines the direction of the spontaneous symmetry breaking. At infinite $N$ the system undergoes at zero field, $h=0$, a secondorder phase transition. At $T=0$ this becomes a quantum phase transition at a value $r_{0}^{c}$ determined from the condition $r=\phi_{0}=0$. Here, we are not interested in this quantum critical point, but in the quantum first-order transition from a state with $\phi_{0}$ to $-\phi_{0}$ that takes place when $h$ changes its sign for $r_{0}<r_{0}^{c}$. The finite-size scaling analysis of the above model was worked out in Ref. [74]. Following this analysis for $\alpha>1$, we obtain for the field dependence of the order parameter at finite $N$,

$$
\phi_{0}(h, N) \sim\left(\frac{r_{0}^{c}-r_{0}}{u}\right)^{\frac{\alpha}{\alpha-1}} h N^{\frac{\alpha}{\alpha-1}} .
$$

For $\alpha=1$, we obtain no longer a power-law relation but an exponential dependence of the order parameter on $N$ :

$$
\phi_{0}=\frac{h}{4 \pi} N^{2 / d} \exp \left(\pi \frac{r_{0}^{c}-r_{0}}{u} N\right) .
$$

The reason is that at $T=0$ a finite system is located at the lower critical dimension [74]. For $\alpha<1$, even the finite system is allowed to order in the ground state, an effect caused through the coupling to an infinite bath that gives rise to the sub-Ohmic dynamics. Then a finite-size analysis will not be the natural approach to soften the first-order quantum phase transition and the expansion Eq. (A7) is no longer justified. In what follows, we focus on $\alpha \geqslant 1$. Comparing Eq. (A14) with Eq. (A7), we obtain for the dynamic scaling exponent at the discontinuity fixed point

$$
z=\frac{d}{\alpha-1} .
$$

This result for $z$ is fundamentally distinct from the one that governs the quantum critical point, where $z_{\text {q.c. }}=2 / \alpha$ [76-79]. At the quantum DFP, even a diverging exponent $z$ is possible. Our next example will illustrate the underlying physics further.

\section{Fully connected quantum rotor model}

Since the SYK model discussed in the main text is a fully connected or infinite-range model, we next discuss the DFP of a fully connected model of $M$-component quantum rotors with $\phi_{i}^{2}(\tau)=1$. We will not take the large- $M$ limit. The action of our model is

$$
S=-\frac{J}{N} \int_{0}^{\beta} d \tau \sum_{i, j=1}^{N} \boldsymbol{\phi}_{i}(\tau) \cdot \boldsymbol{\phi}_{j}(\tau)+\sum_{i=1}^{N} S_{\mathrm{dyn}}\left[\boldsymbol{\phi}_{i}\right] .
$$

The interaction is between all pairs of sites $i$ and $j$. The dynamics is again governed by the exponent $\alpha$,

$$
S_{\mathrm{dyn}}\left[\boldsymbol{\phi}_{i}\right]=\gamma T \sum_{n=-\infty}^{\infty}\left|\omega_{n}\right|^{\alpha} \boldsymbol{\phi}_{i}\left(\omega_{n}\right) \cdot \boldsymbol{\phi}_{i}\left(-\omega_{n}\right) .
$$

Deep in the ordered state of the infinite system, the scaling behavior of the free energy density can be obtained by following the approach of Ref. [75]. In this case, all spins are aligned in the same direction $\phi(\tau)$ at any given time $\tau$, which yields, up to trivial constants, the effective action

$$
S_{\text {eff }}=N S_{\text {dyn }}[\boldsymbol{\phi}]+h N \int_{0}^{\beta} d \tau \boldsymbol{e}_{h} \cdot \boldsymbol{\phi}(\tau) .
$$

This is a $0+1$ dimensional problem, where the remaining fluctuations are along the time direction. Following Ref. [75], we rescale the imaginary time $\tau \rightarrow \tau / N^{1 /(\alpha-1)}$ which eliminates the factor $N$ in front of $S_{\text {dyn }}$ but yields a new temperature $T \rightarrow T N^{1 /(\alpha-1)}$ and a new field strength $h \rightarrow h N^{\frac{\alpha}{\alpha-1}}$. This yields after a few steps the scaling behavior Eq. (A6) with $z=d /(\alpha-1)$. The order-parameter dependence with system size is according to Eq. (A7)

$$
\phi \sim h N^{1+\frac{z}{d}} \sim h N^{\frac{\alpha}{\alpha-1}},
$$

in full agreement with Eq. (A14) obtained for a finite-range problem in the large- $M$ limit.

These results, together with the underlying $0+1$ dimensional field theory for the order parameter allow for a physically transparent interpretation of the dynamics at a quantum discontinuity fixed point. The energy gap of excitations corresponds to $\Delta \sim N^{-z / d}$. The underlying dynamics is made up of fluctuations of the field between the distinct orderparameter configurations. In other words, the quantum state of the system at finite size is an entangled superposition of the states that eventually form the two states that are separated by the first-order transition.

\section{Transverse-field Ising model}

Our last example is the phase transition in the transversefield Ising model

$$
H=-\frac{J}{N} \sum_{i j} \sigma_{i}^{z} \sigma_{j}^{z}-\Gamma \sum_{i} \sigma_{i}^{x}-h \sum_{i} \sigma_{i}^{z} .
$$

As before, the fact that we use a fully connected model is not important near the DFP. Following the reasoning of the rotor model, we obtain away from the $h=0$ critical point an effective Ising variable $\sigma^{z}(\tau)$ that at any given time characterizes the two ordered states

$$
|\uparrow\rangle=|\uparrow \uparrow \uparrow \cdots \uparrow\rangle \quad \text { and } \quad|\Downarrow\rangle=|\downarrow \downarrow \downarrow \cdots \downarrow\rangle
$$


of the entire system and that can be described by the action

$$
S_{\mathrm{eff}}=\frac{N}{\Gamma} \int_{0}^{\beta} d \tau\left(\frac{\partial \sigma^{z}(\tau)}{\partial \tau}\right)^{2}+h N \int_{0}^{\beta} d \tau \sigma^{z}(\tau) .
$$

Again, for finite systems transitions between the two states are possible. The model corresponds to a one-dimensional Ising model along the imaginary time direction and with exchange coupling $N / \Gamma$, i.e., for $T \ll \Delta$ the characteristic excitation energy $\Delta \sim e^{-a N}$ is exponentially small. It is now more convenient to introduce $b \rightarrow b^{d+z}$. Then, Eq. (A6) becomes

$$
f(h, T, N)=b^{-1} f\left(b h, b^{\varphi} T, b^{1-\varphi} N\right),
$$

with $\varphi=z /(d+z)$. The appropriate scaling theory of the transverse-field Ising model corresponds to the limit $\varphi \rightarrow 1$, which amounts to $z / d \rightarrow \infty$. The exponent $1-\varphi$ for the particle size then transforms into logarithmic scaling

$$
f(h, T, N)=b^{-1} f\left(b h, b T, N-\frac{1}{a} \ln b\right),
$$

with nonuniversal coefficient $a$. Using the scaling condition $N-\frac{1}{a} \ln b=1$ we obtain at $T=0$ and $N \gg 1$ for the order parameter $\phi(h, N)=\phi\left(h e^{a N}, 1\right)$ which gives rise to an exponentially large susceptibility, i.e.,

$$
\phi(h, N) \sim h e^{a N}=\frac{h}{\Delta} .
$$

Here $\Delta$ is the tunneling rate between the two ordered states $|\Uparrow\rangle$ and $|\Downarrow\rangle$. Hence, the entire system is really in the state

$$
|\Psi\rangle=\frac{1}{\sqrt{2}}(|\Uparrow\rangle-|\Downarrow\rangle)
$$

again demonstrating that the flow towards a quantum discontinuity fixed point describes the entanglement between the two ordered states that are separated by the first-order transition in the infinite-size limit. $\Delta$ grows exponentially with the system size, just like the rotor problem at $\alpha=1$ of Eq. (A15), because the one-dimensional model in the time direction is in both cases at its lower critical dimension.

\section{Relevance for the DFP in the SYK model}

In analogy to the persistence length scale $\xi_{p}$ discussed for classical transitions in Ref. [56], a quantum discontinuity point is governed by a persistence timescale $\tau_{p} \sim N^{z / d}$ or $\tau_{p} \sim \exp (a N)$. Its divergence corresponds to long-time order in correlation functions that distinguish the two phases, i.e., the tunneling rate between both phases. For the SYK model, a finite-size scaling analysis as discussed here is problematic because the large- $N$ limit does not commute with the low-temperature limit [61]. However, the transition can be softened by means of other external parameters [35], as mentioned at the end of subsection 1 . This opens the possibility to probe the scaling exponent and persistence timescale at the phase transition of the SYK model and with that the anomalous dimension at the DFP.

\section{APPENDIX B: TWO-PARTICLE RESPONSE IN THE INTEGER VALENCE PHASE}

In this Appendix, we summarize the analysis that leads to Eq. (61) for the two-particle vertex of the integer valence phase. Without restriction, we consider $\mu<0$ so that the ground state is empty and a single electron added to the system has no other electron to interact with. This does not apply to higher-particle correlations, where one adds two or more particles and follows their evolution in time. As shown by Galitskii [62], the two-particle vertex of a such a dilute system can be obtained by summing up all particle-particle ladder diagrams. We first determine the two-particle vertex for a given disorder configuration. The disorder average will then be performed at the end.

In the integer valence state we can use the fact that at $T=0$ the single-particle spectrum is trivial. Hence, the propagator is

$$
G_{i j}(\omega)=\frac{\delta_{i j}}{i \omega+\mu} .
$$

Next we consider the two-particle response. We express the fully renormalized four-particle vertex as

$$
\begin{aligned}
\gamma_{i j k l}\left(\omega_{1}, \omega_{2}, \omega_{3}, \omega_{4}\right)= & \Gamma_{i j k l}\left(\omega_{1}, \omega_{2} ; \frac{\omega_{4}-\omega_{3}}{2}\right) \\
& \times \delta\left(\omega_{1}+\omega_{2}-\omega_{3}-\omega_{4}\right),
\end{aligned}
$$

which respects energy conservation. At low density, it suffices to sum particle-particle diagrams. Then one finds that the vertex only depends on $\Omega \equiv \omega_{1}+\omega_{2}$. This leads to

$$
\Gamma_{i j k l}(\Omega)=J_{i j k l}-\sum_{m<n} \int_{\omega^{\prime}} J_{i j m n} \Gamma_{m n k l}(\Omega) \chi(\Omega),
$$

where we introduced the particle-particle bubble,

$$
\begin{aligned}
\chi\left(\omega_{1}+\omega_{2}\right) & =\int_{\omega^{\prime}} G\left(\omega_{1}-\omega^{\prime}\right) G\left(\omega_{2}+\omega^{\prime}\right) \\
& =\frac{\operatorname{sign}(\mu)}{i \omega_{1}+i \omega_{2}+2 \mu} .
\end{aligned}
$$

It is convenient to introduce double indices $a=(i, j)$ and $b=$ $(k, l)$ for two-particle states and rewrite Eq. (B3) as

$$
\Gamma_{a b}(\Omega)=J_{a b}-\sum_{c} J_{a c} \Gamma_{c b}(\Omega) \chi(\Omega) .
$$

Here $c=(m, n)$ with $m<n$ runs over $M=\frac{1}{2} N(N-1)$ distinct values. This matrix equation gives rise to the formal solution

$$
\begin{aligned}
\hat{\Gamma}(\Omega) & =[1+\hat{J} \chi(\Omega)]^{-1} \hat{J} \\
& =\sum_{n=1}^{\infty}(-1)^{n-1} \hat{J}^{n}[\chi(\Omega)]^{n-1},
\end{aligned}
$$

where $[\hat{J}]_{a b}=J_{a b}$. Next we perform the average of the vertex function over the random configurations $J_{a b}$ using

$$
\overline{J_{a b} J_{a^{\prime} b^{\prime}}}=\overline{J_{a b} J_{b^{\prime} a^{\prime}}^{*}}=\frac{2 J^{2}}{N^{3}} \delta_{a b^{\prime}} \delta_{b a^{\prime}},
$$




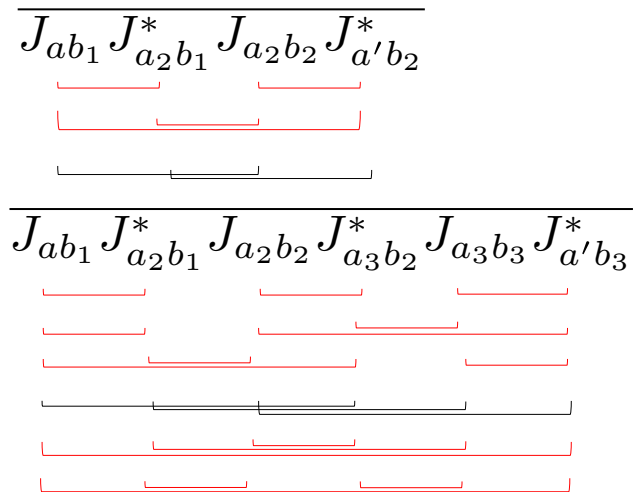

FIG. 10. Contractions that occur when one averages the twoparticle vertex over the disorder configurations. In the upper part all three contractions at order $\mathcal{O}\left(J^{4}\right)$ are given. Crossed diagrams (black) are of sub-leading order in $1 / N$ and can be neglected. Only noncrossing contractions (red) contribute at large $N$. In the lower part we focus on the 3 ! contractions between $J_{\mathrm{s}}$ and $J^{*} s$ at order $\mathcal{O}\left(J^{6}\right)$. Of those one is also a crossing diagram (black) and can be neglected as well. Hence five noncrossing diagrams contribute at large $N$ (red).

see also Eq. (2). All terms with odd $n$ vanish. The secondorder contribution is

$$
\Gamma_{a b}^{(2)}(\Omega)=-\sum_{c} J_{a c} J_{c b} \chi(\Omega) .
$$

Performing the average gives

$$
\overline{\Gamma_{a a^{\prime}}^{(2)}(\Omega)}=-\sum_{b} \overline{J_{a b} J_{a^{\prime} b}^{*}} \chi(\Omega)=-\frac{J^{2} \delta_{a a^{\prime}}}{N} \chi(\Omega),
$$

where we used $M \approx N^{2} / 2$ at large- $N$. At fourth order we have

$$
\Gamma_{a a^{\prime}}^{(4)}(\Omega)=-\sum_{b_{1} b_{2} a_{2}} J_{a b_{1}} J_{b_{1} a_{2}} J_{a_{2} b_{2}} J_{b_{2} a^{\prime}}[\chi(\Omega)]^{3} .
$$

To average this expression over disorder, we use Wick's theorem,

$$
\begin{aligned}
\overline{J_{a b_{1}} J_{b_{1} a_{2}} J_{a_{2} b_{2}} J_{b_{2} a^{\prime}}}= & \overline{J_{a b_{1}} J_{a_{2} b_{1}}^{*} J_{a_{2} b_{2}} J_{a^{\prime} b_{2}}^{*}} \\
= & \left(\frac{2 J^{2}}{N^{3}}\right)^{2}\left(\delta_{a a_{2}} \delta_{a_{2} a^{\prime}}+\delta_{a a^{\prime}} \delta_{b_{1} b_{2}}\right. \\
& \left.+\delta_{a b_{2}} \delta_{b_{1} a_{2}} \delta_{b_{1} a^{\prime}} \delta_{a_{2} b_{2}}\right) .
\end{aligned}
$$

At large $N$ this gives rise to

$$
\overline{\Gamma_{a a^{\prime}}^{(4)}(\Omega)}=-2 \delta_{a a^{\prime}}\left(\frac{J^{2}}{N}\right)^{2}[\chi(\Omega)]^{3} .
$$

Here, the prefactor 2 is the result of the first two contractions that occur in Eq. (B11). The last contraction can be neglected at large $N$. Diagrammatically, it corresponds to crossed lines, see Fig. 10. To get rid of many of such crossed contractions, we can focus on only contracting indices at even positions with those at odd positions. This is simplified by considering the complex conjugation for the even positions, see Eq. (B11). At order $n=6$ we have to analyze

$$
\overline{J_{a b_{1}} J_{a_{2} b_{1}}^{*} J_{a_{2} b_{2}} J_{a_{3} b_{2}}^{*} J_{a_{3} b_{3}} J_{a^{\prime} b_{3}}^{*}} .
$$

In total there are $\frac{n}{2} !=3 !=6$ contractions between even and odd positions (between $J_{\mathrm{s}}$ and $J^{*} \mathrm{~s}$ ). However, not all have the same relevance at large- $N$. One contraction occurs with a crossed line and is negligible at large $N$, see Fig. 10. It then follows:

$$
\overline{\Gamma_{a a^{\prime}}^{(6)}(\Omega)}=-5 \delta_{a a^{\prime}}\left(\frac{J^{2}}{N}\right)^{3}[\chi(\Omega)]^{5} .
$$

Let us now discuss the contribution at order $n=2 p$, which we write as

$$
\overline{\Gamma_{a a^{\prime}}^{(2 p)}(\Omega)}=-C_{p} \delta_{a a^{\prime}}\left(\frac{J^{2}}{N}\right)^{p}[\chi(\Omega)]^{2 p-1},
$$

where $C_{p}$ is the number of contractions without crossing. In combinatorial mathematics this is called the problem of proper parenthesization and is given by the Catalan numbers [81],

$$
C_{p}=\frac{1}{2 p+1}\left(\begin{array}{c}
2 p \\
p
\end{array}\right) .
$$

Indeed, $C_{1}=1, C_{2}=2, C_{3}=5$ as was obtained in the explicit analysis. Now we can sum the entire series and obtain

$$
\overline{\Gamma_{a a^{\prime}}(\Omega)}=-\delta_{a a^{\prime}} \sum_{p=1}^{\infty} C_{p}\left(\frac{J^{2}}{N}\right)^{p}[\chi(\Omega)]^{2 p-1} .
$$

Finally, using

$$
\sum_{p=1}^{\infty} C_{p} x^{p}=\frac{2}{1+\sqrt{1-4 x}}-1
$$

for the generating function of the Catalan numbers we obtain the following result for the averaged four-particle vertex in the integer-valence phase,

$$
\overline{\Gamma_{a a^{\prime}}(\Omega)}=-\delta_{a a^{\prime}}(i \Omega+2 \mu)\left(1-\frac{2}{1+\sqrt{1-\frac{4 J^{2}}{N(i \Omega+2 \mu)^{2}}}}\right) .
$$

Analytic continuation to the real frequency axis yields the result given in Eq. (61).

\section{APPENDIX C: LARGE- $N$ SCHWINGER-DYSON EQUATIONS WITH INFRARED CUTOFF}

In this Appendix, we outline our numerical methods to extract the large- $N$ thermodynamics of the NFL and DFP phases from the Schwinger-Dyson equations (59) for the scale-dependent dressed fermionic propagator (19). Analogously to other self-consistent loops for SYK self-energies [34-39,43,44], we use fast Fourier transform (FFT) to switch between the representations of the propagator in discretized imaginary time $\tau_{l}=l /\left(2 N-f k_{B} T\right)$ with $l \in[0,2 N-f-$ 1] and Matsubara frequencies $\omega_{n}=(2 n+1) \pi k_{B} T$ with $n \in$ $\left[-N_{f}, N_{f}-1\right]$, as implemented by the Fourier [] and InverseFourier [] built-in functions of MATHEMATICA. To analyze the NFL solution, the initial condition for the selfconsistent loop (59) is set to the low-energy expression (5). Alternatively, any initial guess for $\Sigma\left(i \omega_{n}\right)$ deemed close to the actual solution, i.e., a previously found solution at a slightly 


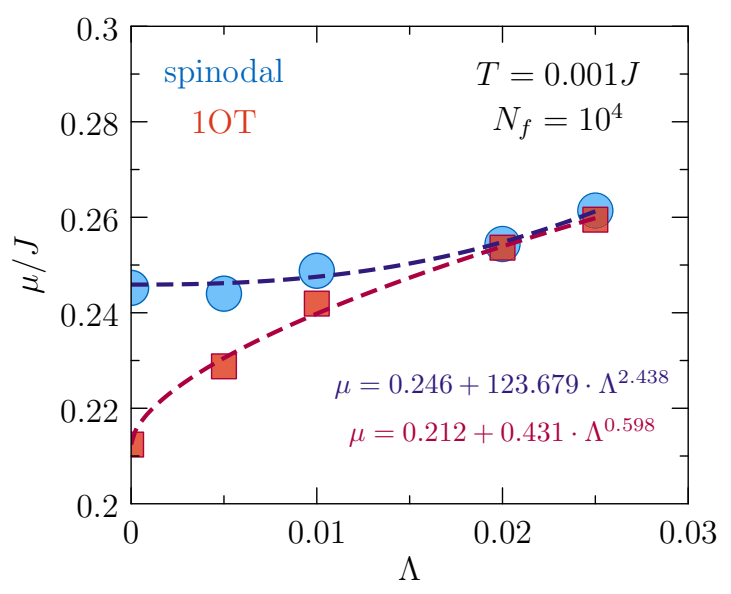

FIG. 11. Chemical potential corresponding to the 1OT line (red squares) and for the rightmost spinodal in Fig. 2 (light blue circles) as a function of infrared cutoff $\Lambda$.

different value of $\mu$, can be used. Instead, convergence to the IV solution is facilitated by setting the initial condition $\Sigma_{\Lambda}(\omega)=0$ for all Matsubara frequencies $\omega$.

The fermion density is

$$
n_{\Lambda}=\left.\frac{1}{\beta} \sum_{\omega} G_{\Lambda}(\omega) e^{-i \omega 0^{+}} \equiv G_{\Lambda}(\tau)\right|_{\tau \rightarrow 0^{-}},
$$

which can be rewritten as

$$
n_{\Lambda}=\frac{1}{e^{-\beta \mu}+1}+\frac{1}{\beta} \sum_{\omega} \operatorname{Re}\left\{G_{\Lambda}(\omega)-G_{0}(\omega)\right\}
$$

by adding and subtracting the free-fermion contribution stemming from $G_{0}(\omega)=(i \omega-\mu)^{-1}$.

Using the self-consistent $G_{\Lambda}(\omega)$ and $\Sigma_{\Lambda}(\omega)$, we obtain the grand potential from

$$
\begin{aligned}
\frac{\Omega_{\Lambda}}{N}= & \beta^{-1} \sum_{\omega} \ln \left[\frac{G_{\Lambda}(\omega)}{G_{0}(\omega)}\right]-\frac{3}{4} \beta^{-1} \sum_{\omega} \Sigma_{\Lambda}(\omega) G_{\Lambda}(\omega) \\
& -\beta^{-1} \ln \left(1+e^{\beta \mu}\right),
\end{aligned}
$$

where again the free-fermion contribution has been added and subtracted to ease the convergence of the Matsubara sums.

The difference between the grand potentials of the NFL and IV solutions of Eq. (C3), namely $\Omega_{\Lambda}^{\mathrm{NFL}}$ and $\Omega_{\Lambda}^{\mathrm{IV}}$, is

$$
\frac{\Delta \Omega(\Lambda)}{N}=\frac{\Omega_{\Lambda}^{\mathrm{NFL}}}{N}-\frac{\Omega_{\Lambda}^{\mathrm{IV}}}{N}
$$

The condition $\Delta \Omega(\Lambda)=0$ is evaluated numerically, to find the first-order phase transition (1OT) point in the $(\mu, T)$ diagram for a given cutoff $\Lambda$. The result for the critical chemical potential $\mu_{c}(\Lambda)$ at temperature $T=0.001 \mathrm{~J}$ is displayed by the red squares in Fig. 11. The same figure shows the chemical potential at the rightmost spinodal line $\mu_{*}(\Lambda)$ as blue circles. The dashed red and blue curves are fits of the numerical data for the 1OT and spinodal lines, respectively.

Figure 7 shows the fermion density $n(\mu)$ for different cutoffs $\Lambda>0$ : in the top panel, we see that a finite $\Lambda$ weakens the 1 OT by reducing the size of the density discontinuity at the transition, and that for $\Lambda>\Lambda_{T} \approx 0.025 J$ the transition is completely smeared out into a continuous $n(\mu)$ curve. This value $\Lambda_{T}$ corresponds to the cutoff at which the 1OT and the spinodal merge, as seen in Fig. 11. Such smearing of the 1OT is reminiscent of a finite-size scaling analysis of the kinds described in Appendix A, where now the energy $\Lambda$ plays the role of the size $N$. The existence of a threshold $\Lambda_{T}$ may reflect the requirement of overcoming the energy barrier between the NFL and IV fixed points, which would translate into a finite threshold $\Delta$ to destabilize the corresponding fixed points in the $T=0$ FRG flow.

We numerically calculate the self-energy $\Sigma_{\Lambda}^{*}(\omega)$ at $\mu=$ $\mu_{*}$ and given $\Lambda$. Such self-energy is defined at the discrete set of Matsubara frequencies, but its low-energy real part approaches a constant value. By fitting $\operatorname{Re} \Sigma_{\Lambda}^{*}(\omega)$ at low Matsubara frequencies $\omega$ and extrapolating the fitted function to zero frequency, we obtain the values of $\operatorname{Re} \Sigma(0) \equiv \Sigma(0)$ shown in Fig. 8.
[1] J. D. van der Waals, Over de continuiteit van den gas - en vloeistoftoestand, Ph.D. thesis (A. W. Sijthoff, Leiden, 1873).

[2] M. A. Stephanov, QCD phase diagram and the critical point, Prog. Theor. Phys. Suppl. 153, 139 (2004).

[3] T. Tanaka, Collapse of Gels and the Critical Endpoint, Phys. Rev. Lett. 40, 820 (1978).

[4] N. F. Mott, Metal-Insulator Transition (WILEY-VCH Verlag, Weinheim, 1990).

[5] C. Castellani, C. Di Castro, D. Feinberg, and J. Ranninger, New Model Hamiltonian for the Metal-Insulator Transition, Phys. Rev. Lett. 43, 1957 (1979).

[6] G. Kotliar, E. Lange, and M. J. Rozenberg, Landau Theory of the Finite Temperature Mott Transition, Phys. Rev. Lett. 84, 5180 (2000).

[7] P. Majumdar and H. R. Krishnamurthy, Lattice Contraction Driven Insulator-Metal Transition in the $d=\infty$ Local Approximation, Phys. Rev. Lett. 73, 1525 (1994).
[8] P. Limelette, A. Georges, D. Jérome, P. Wzietek, P. Metcalfa, and J. M. Honig, Universality and critical behavior at the Mott transition, Science 302, 89 (2003).

[9] S. R. Hassan, A. Georges, and H. R. Krishnamurthy, Sound Velocity Anomaly at the Mott Transition: Application to Organic Conductors and $\mathrm{V}_{2} \mathrm{O}_{3}$, Phys. Rev. Lett. 94, 036402 (2005).

[10] S. Lefebvre, P. Wzietek, S. Brown, C. Bourbonnais, D. Jérome, C. Mézière, M. Fourmigué, and P. Batail, Mott Transition, Antiferromagnetism, and Unconventional Superconductivity in Layered Organic Superconductors, Phys. Rev. Lett. 85, 5420 (2000).

[11] F. Kagawa, K. Miyagawa, and K. Kanoda, Magnetic Mott criticality in a $\kappa$-type organic salt probed by NMR, Nature (London) 436, 534 (2005).

[12] M. de Souza, A. Brühl, C. Strack, B. Wolf, D. Schweitzer, and M. Lang, Anomalous Lattice Response at the Mott Transition 
in a Quasi-2D Organic Conductor, Phys. Rev. Lett. 99, 037003 (2007).

[13] L. Bartosch, M. de Souza, and M. Lang, Scaling Theory of the Mott Transition and Breakdown of the Grüneisen Scaling Near a Finite-Temperature Critical End Point, Phys. Rev. Lett. 104, 245701 (2010).

[14] E. Gati, M. Garst, R. S. Manna, U. Tutsch, B. Wolf, L. Bartosch, H. Schubert, T. Sasaki, J. A. Schlueter, and M. Lang, Breakdown of Hooke's law of elasticity at the Mott critical endpoint in an organic conductor, Sci. Adv. 2, e1601646 (2016).

[15] A. Pustogow, M. Bories, A. Löhle, R. Rösslhuber, E. Zhukova, B. Gorshunov, S. Tomić, J. A. Schlueter, R. Hübner, T. Hiramatsu, Y. Yoshida, G. Saito, R. Kato, T.-H. Lee, V. Dobrosavljević, S. Fratini, and M. Dressel, Quantum spin liquids unveil the genuine Mott state, Nat. Mater. 17, 773 (2018).

[16] A. Pustogow, R. Rösslhuber, Y. Tan, E. Uykur, M. Wenzel, A. Böhme, A. Löhle, R. Hübner, Y. Saito, A. Kawamoto, J. A. Schlueter, V. Dobrosavljević, and M. Dressel, Low-temperature dielectric anomalies at the Mott insulator-metal transition, npj Quantum Mater. 6, 9 (2021).

[17] R. Rösslhuber, A. Pustogow, E. Uykur, A. Böhme, A. Löhle, R. Hübner, J. Schlueter, Y. Tan, V. Dobrosavljević, and M. Dressel, Phase coexistence at the first-order Mott-transition revealed by pressure-dependent dielectric spectroscopy of $\kappa-($ BEDTTTF $)_{2} \mathrm{Cu}_{2}(\mathrm{CN})_{3}$, Phys. Rev. B 103, 125111 (2021).

[18] J. M. Lawrence, P. S. Riseborough, and R. D. Parks, Valence fluctuation phenomena, Rep. Prog. Phys. 44, 1 (1981).

[19] J. L. Sarrao, Physics of $\mathrm{YbInCu}_{4}$ and related compounds, Physica B (Amsterdam) 259-261, 128 (1999).

[20] J. W. Allen and R. M. Martin, Kondo Volume Collapse and the $\gamma \rightarrow \alpha$ Transition in Cerium, Phys. Rev. Lett. 49, 1106 (1982).

[21] M. Dzero, M. R. Norman, I. Paul, C. Pépin, and J. Schmalian, Quantum Critical End Point for the Kondo Volume Collapse Model, Phys. Rev. Lett. 97, 185701 (2006); Erratum: Quantum Critical End Point for the Kondo Volume Collapse Model [Phys. Rev. Lett. 97, 185701 (2006)] Phys. Rev. Lett. 104, 119901 (2010).

[22] N. Lanatà, Y.-X. Yao, C.-Z. Wang, K.-M. Ho, J. Schmalian, K. Haule, and G. Kotliar, $\gamma-\alpha$ Isostructural Transition in Cerium, Phys. Rev. Lett. 111, 196801 (2013).

[23] F. Honda, K. Okauchi, A. Nakamura, D. Li, D. Aoki, H. Akamine, Y. Ashitomi, M. Hedo, T. Nakama, and Y. Onuki, Pressure-Induced Valence Transition and Characteristic Electronic States in $\mathrm{EuRh}_{2} \mathrm{Si}_{2}$, J. Phys. Soc. Jpn. 85, 063701 (2016).

[24] A. P. Levanyuk and A. A. Sobyanin, Second-order phase transitions without divergences in the second order derivatives of the thermodynamic potential, Zh. Eksp. Teor. Fiz. Pis. Red. 11, 540 (1970).

[25] A. Hackl and M. Vojta, Kondo volume collapse, Kondo breakdown, and Fermi surface transitions in heavy-fermion metals, Phys. Rev. B 77, 134439 (2008).

[26] S. Papanikolaou, R. M. Fernandes, E. Fradkin, P. W. Phillips, J. Schmalian, and R. Sknepnek, Universality of Liquid-Gas Mott Transitions at Finite Temperatures, Phys. Rev. Lett. 100, 026408 (2008).

[27] M. Zacharias, L. Bartosch, and M. Garst, Mott Metal-Insulator Transition on Compressible Lattices, Phys. Rev. Lett. 109, 176401 (2012).
[28] M. Zacharias, A. Rosch, and M. Garst, Critical elasticity at zero and finite temperature, Eur. Phys. J. Spec. Top. 224, 1021 (2015).

[29] C. Pfleiderer, Why first order quantum phase transitions are interesting, J. Phys.: Condens. Matter 17, S987 (2005).

[30] T. Jörg, F. Krzakala, J. Kurchan, A. C. Maggs, and J. Pujos, Energy gaps in quantum first-order mean-field-like transitions: The problems that quantum annealing cannot solve, Europhys. Lett. 89, 40004 (2010).

[31] C. R. Laumann, R. Moessner, A. Scardicchio, and S. L. Sondhi, Quantum Adiabatic Algorithm and Scaling of Gaps at FirstOrder Quantum Phase Transitions, Phys. Rev. Lett. 109, 030502 (2012).

[32] B. Zhao, P. Weinberg, and A. W. Sandvik, Symmetry-enhanced discontinuous phase transition in a two-dimensional quantum magnet, Nat. Phys. 15, 678 (2019).

[33] S. Banerjee and E. Altman, Solvable model for a dynamical quantum phase transition from fast to slow scrambling, Phys. Rev. B 95, 134302 (2017).

[34] T. Azeyanagi, F. Ferrari, and F. I. Schaposnik Massolo, Phase Diagram of Planar Matrix Quantum Mechanics, Tensor, and Sachdev-Ye-Kitaev Models, Phys. Rev. Lett. 120, 061602 (2018).

[35] A. A. Patel and S. Sachdev, Theory of a Planckian Metal, Phys. Rev. Lett. 123, 066601 (2019).

[36] Y. Wang and A. V. Chubukov, Quantum phase transition in the Yukawa-SYK model, Phys. Rev. Res. 2, 033084 (2020).

[37] F. Ferrari and F. I. Schaposnik Massolo, Phases of melonic quantum mechanics, Phys. Rev. D 100, 026007 (2019).

[38] N. Sorokhaibam, Phase transition and chaos in charged SYK model, J. High Energy Phys. 07 (2020) 055.

[39] S. Sachdev and J. Ye, Gapless Spin-Fluid Ground State in a Random Quantum Heisenberg Magnet, Phys. Rev. Lett. 70, 3339 (1993).

[40] A. Y. Kitaev, A Simple Model of Quantum Hologarphy, Talks at KITP, University of California, Santa Barbara, April 7, 2015 and May 27, 2015, the recording is available at https://online. kitp.ucsb.edu/online/entangled15/kitaev/ (part 1) and https:// online.kitp.ucsb.edu/online/entangled15/kitaev2/ (part 2).

[41] S. Sachdev, Bekenstein-Hawking Entropy and Strange Metals, Phys. Rev. X 5, 041025 (2015).

[42] J. Polchinski and V. Rosenhaus, The spectrum of the SachdevYe-Kitaev model, J. High Energy Phys. 04 (2016) 001.

[43] J. Maldacena and D. Stanford, Remarks on the Sachdev-YeKitaev model, Phys. Rev. D 94, 106002 (2016).

[44] X.-Y. Song, C.-M. Jian, and L. Balents, Strongly Correlated Metal Built from Sachdev-Ye-Kitaev Models, Phys. Rev. Lett. 119, 216601 (2017).

[45] V. Rosenhaus, An introduction to the SYK model, J. Phys. A: Math. Theor. 52, 323001 (2019).

[46] S. A. Hartnoll, A. Lucas, and S. Sachdev, Holographic Quantum Matter (The MIT Press, Cambridge, Massachusetts, 2018).

[47] D. A. Trunin, Pedagogical introduction to the SYK model and 2D Dilaton Gravity, Phys. Usp. 64, 219 (2021).

[48] I. Esterlis and J. Schmalian, Cooper pairing of incoherent electrons: An electron-phonon version of the Sachdev-Ye-Kitaev model, Phys. Rev. B 100, 115132 (2019).

[49] Y. Wang, Solvable Strong-Coupling Quantum-Dot Model with a Non-Fermi-Liquid Pairing Transition, Phys. Rev. Lett. 124, 017002 (2020). 
[50] M. Salmhofer and C. Honerkamp, Fermionic renormalization group flows, Prog. Theor. Phys. 105, 1 (2001).

[51] P. Kopietz and T. Busche, Exact renormalization group flow equations for nonrelativistic fermions: Scaling toward the Fermi surface, Phys. Rev. B 64, 155101 (2001).

[52] P. Kopietz, L. Bartosch, and F. Schütz, Introduction to the Functional Renormalization Group (Springer, Berlin, 2010).

[53] W. Metzner, M. Salmhofer, C. Honerkamp, V. Meden, and K. Schönhammer, Functional renormalization group approach to correlated fermion systems, Rev. Mod. Phys. 84, 299 (2012).

[54] N. Dupuis, L. Canet, A. Eichhorn, W. Metzner, J. M. Pawlowski, M. Tissier, and N. Wschebor, The nonperturbative functional renormalization group and its applications, Phys. Rep. 910, 1 (2021).

[55] B. Nienhuis and M. Nauenberg, First-Order Phase Transitions in Renormalization-Group Theory, Phys. Rev. Lett. 35, 477 (1975).

[56] M. E. Fisher and A. N. Berker, Scaling for first-order phase transitions in thermodynamic and finite systems, Phys. Rev. B 26, 2507 (1982).

[57] Frequently, the SYK literature uses the exponent $\Delta=\eta / 2$.

[58] C. Wetterich, Exact evolution equation for the effective potential, Phys. Lett. B 301, 90 (1993).

[59] T. R. Morris, The exact renormalisation group and approximate solutions, Int. J. Mod. Phys. A 9, 2411 (1994)

[60] A. A. Katanin, Fulfillment of Ward identities in the functional renormalization group approach, Phys. Rev. B 70, 115109 (2004).

[61] D. Bagrets, A. Altland, and A. Kamenev, Sachdev-Ye-Kitaev model as Liouville quantum mechanics, Nucl. Phys. B 911, 191 (2016).

[62] V. M. Galitskii, The energy spectrum of a non-ideal Fermi gas, Zh. Eksp. Teor. Fiz. 34, 151 (1957) [Sov. Phys. JETP 34, 104 (1958)].

[63] S. Sahoo, E. Lantagne-Hurtubise, S. Plugge, and M. Franz, Traversable wormhole and Hawking-Page transition in coupled complex SYK models, Phys. Rev. Res. 2, 043049 (2020).

[64] I. R. Klebanov, A. Milekhin, and G. Tarnopolsky, Spontaneous breaking of U(1) symmetry in coupled complex SYK models, J. High Energy Phys. 11 (2020) 162.

[65] C. Borgs and R. Kotecky, Finite-Size Effects at Asymmetric First-Order Phase Transitions, Phys. Rev. Lett. 68, 1734 (1992).
[66] Y. C. Kim, M. E. Fisher, and G. Orkoulas, Asymmetric fluid criticality. I. Scaling with pressure mixing, Phys. Rev. E 67, 061506 (2003).

[67] V. Privman and J. Rudnick, Nonsymmetric first-order transitions: Finite-size scaling and tests for infinite-range models, J. Stat. Phys. 60, 551 (1990).

[68] Finite Size Scaling and Numerical Simulation of Statistical Systems, edited by V. Privman (World Scientific, Singapore, 1990).

[69] F. Igloi and L. Turban, First-order line transitions: A general scaling theory with irrelevant variables, Phys. Rev. B 47, 3404 (1993).

[70] J. Solyom and P. Pfeuty, Renormalization-group study of the Hamiltonian version of the Potts model, Phys. Rev. B 24, 218 (1981).

[71] F. Igloi and C. Vanderzande, Renormalization Group Study of the (2+1) dimensional Potts Model, Physica A 135, 347 (1986)

[72] M. A. Continentino and A. S. Ferreira, Quantum first-order phase transitions, Physica A 339, 461 (2004).

[73] A. S. Ferreira and M. A. Continentino, Universal behaviour at discontinuous quantum phase transitions, J. Stat. Mech. (2005) P05005.

[74] T. Vojta and J. Schmalian, Quantum Griffiths effects in itinerant Heisenberg magnets, Phys. Rev. B 72, 045438 (2005).

[75] T. Vojta and J. Schmalian, Percolation Quantum Phase Transitions in Diluted Magnets, Phys. Rev. Lett. 95, 237206 (2005).

[76] A. V. Chubukov, S. Sachdev, and J. Ye, Theory of twodimensional quantum Heisenberg antiferromagnets with a nearly critical ground state, Phys. Rev. B 49, 11919 (1994).

[77] J. A. Hertz, Quantum critical phenomena, Phys. Rev. B 14, 1165 (1976).

[78] A. J. Millis, Effect of a nonzero temperature on quantum critical points in itinerant fermion systems, Phys. Rev. B 48, 7183 (1993).

[79] S. Sachdev, A. V. Chubukov, and A. Sokol, Crossover and scaling in a nearly antiferromagnetic Fermi liquid in two dimensions, Phys. Rev. B 51, 14874 (1995).

[80] P. Gagel, P. P. Orth, and J. Schmalian, Universal post-quench coarsening and aging at a quantum critical point, Phys. Rev. B 92, 115121 (2015).

[81] R. P. Stanley, Enumerative Combinatorics (Cambridge University Press, Cambridge, 1999), Vol. 2. 